\title{
Spatial variations of the optical galaxy luminosity functions and red sequences in the Coma cluster: clues to its assembly history $\star, \star \star$
}

\author{
C. Adami ${ }^{1}$, F. Durret ${ }^{2,3}$, A. Mazure ${ }^{1}$, R. Pelló ${ }^{4}$, J. P. Picat ${ }^{4}$, M. West ${ }^{5}$, and B. Meneux ${ }^{1,6,7}$ \\ 1 LAM, Traverse du Siphon, 13012 Marseille, France \\ e-mail: christophe.adami@oamp. fr \\ 2 Institut d'Astrophysique de Paris, CNRS, Université Pierre et Marie Curie, 98bis Bd Arago, 75014 Paris, France \\ 3 Observatoire de Paris, LERMA, 61 Av. de l'Observatoire, 75014 Paris, France \\ 4 Observatoire Midi-Pyrénées, 14 Av. Edouard Belin, 31400 Toulouse, France \\ 5 Department of Physics and Astronomy, University of Hawaii, 200 West Kawili Street, LS2, Hilo HI 96720-4091, USA \\ 6 INAF - IASF, via Bassini 15, 20133 Milano, Italy \\ 7 INAF, Osservatorio Astronomico di Brera, via Bianchi 46, 23807 Merate (LC), Italy
}

Accepted 16 June 2006 / Accepted 13 September 2006

\section{ABSTRACT}

\begin{abstract}
Context. Clusters of galaxies are believed to be at the intersections of cosmological filaments and to grow by accreting matter from these filaments. Such continuous infall has major consequences not only on clusters, but also on the physics of cluster galaxies. Faint galaxies are particularly interesting as they are very sensitive to environmental effects and may have different behavior from those of bright galaxies.

Aims. The aim of this paper is to sample the Coma cluster building history, based on the analysis of galaxy luminosity functions (LFs) and red sequences (RSs) in the color magnitude relation (CMR) down to faint magnitudes, which are privileged tools for this purpose. Methods. The present analysis is based on deep $(R \sim 24)$, wide $\left(\sim 0.5 \mathrm{deg}^{2}\right)$ multiband (BVRI Vega system) images of the Coma cluster obtained with the CFH12K camera at the CFHT. We have derived LFs and CMRs in twenty $10 \times 10 \operatorname{arcmin}^{2}$ regions and in larger regions.

Results. In all photometric bands, we found steeply rising LFs in the north-northeast half of the cluster (due to early-type galaxies at bright magnitudes and due to late-type galaxies at the faint end), and much flatter LFs in the south-southwest region. Although the behavior of the CMR RS is different in these two regions, a good agreement is found in general between the RS computed for faint and for bright galaxies.

Conclusions. All these results can be interpreted consistently in the framework of the building up process that has been proposed. The Northern Coma area is a relatively quiescent region, while the southern area experiences several infalls.
\end{abstract}

Key words. galaxies: clusters: individual: Coma

\section{Introduction}

Hierarchical building of clusters is a key ingredient of cosmological models, since clusters are believed to be at the nodes of cosmological filaments and to grow by accreting matter (gas, galaxies, groups, etc.) from filaments (e.g., Lanzoni et al. 2005). Such continuous infall has major consequences on the shape and dynamics of clusters, as well as on the physics of cluster galaxies (e.g., Sarazin 1986), as revealed by the cluster galaxy mass function (usually traced by the luminosity function, hereafter LF) and by the cluster early type galaxy red sequence (hereafter RS). The influence of infall on the LF is relatively well understood for bright galaxies in nearby to intermediate redshift clusters (e.g., Hansen et al. 2005), but only little is known for low-mass galaxies because they are difficult to observe. It is also important to study galaxies at the faint end of the RS because the Color

* Based on observations obtained at the Canada-France-Hawaii Telescope (CFHT) which is operated by the National Research Council of Canada, the Institut National des Sciences de l'Univers of the Centre National de la Recherche Scientifique of France, and the University of Hawaii.

$\star \star$ Figures 10,12, 14 and 18 are only available in electronic form at http://www. aanda.org
Magnitude Relation (hereafter CMR), which traces their star formation history, is directly related to their metal abundances and is poorly known. These faint galaxies are of major interest as their evolutionary paths are sometimes different from those of bright galaxies: they are very sensitive to environmental effects (e.g., fusion with bright galaxies or tidal disruptions), they also keep their dynamical memory longer (e.g., Sarazin 1986), and their spatial distribution is different from that of bright galaxies (e.g., Biviano et al. 1996, but see also Edwards et al. 2002, for a different result)

To study the properties of galaxy clusters through their LF and CMR requires us to sample the whole cluster galaxy populations from the bright $\mathrm{cD}$ to the faintest dwarfs $\left(M_{R} \sim-10\right)$. Very demanding observations are needed both in terms of photometric depth and size of field, and were not possible until the arrival of wide-field facilities such as the CFH12K on the CFHT. With such $4 \mathrm{~m}$-class telescopes, access to the faintest objects is still limited to nearby clusters. In this respect, the Coma cluster is possibly the best target: bright objects are well studied in all wavelengths (e.g., Biviano 1998) and its low redshift allows us to go deep enough towards the faint end of the galaxy population.

LFs and CMRs have recently been computed for bright to intermediate magnitudes in Coma (e.g., Lobo et al. 1997; 
Terlevich et al. 2001; Andreon \& Cuillandre 2002; Beijersbergen et al. 2002; Iglesias-Páramo et al. 2003) in large fields. Deeper studies in rather limited areas also exist (Bernstein et al. 1995; Trentham 1998; or Milne et al. 2006). All these surveys generally have a wavelength coverage that is too limited to provide strong constraints on the Coma cluster building history. For the first time, by using the CFH12K camera at the CFHT, we obtained a set of deep $(R \sim 24)$, wide $\left(\sim 0.5 \mathrm{deg}^{2}\right)$ multiband $(B V R I)$ images of the Coma cluster.

The key to compute a LF or a CMR is to select objects in the cluster and then galaxies, as compared to intergalactic Globular Clusters and galactic stars. For the bright part of our sample, we had enough spectroscopic redshifts to discriminate Coma members. As it is difficult to obtain spectra for the faint part, we used statistical comparisons with empty fields (containing no rich nearby clusters). This is a classical method that requires very homogeneous observations of the cluster and of the empty fields. The empty fields we used were parts of two of the VVDS CFH12K imaging survey fields (McCracken et al. 2003, hereafter MC03) completely re-analyzed so as to have catalogs homogeneous with the Coma data. This allows us to properly treat possible pitfalls, such as diffuse light in the cluster, galactic extinction, differences in the star counts, large-scale structure of the background, and crowding effects. Other problems that could affect the background counts along the Coma line of sight are the presence of dust in the cluster or the gravitational effects on background galaxies, but they have been shown to be negligible in Coma (Bernstein et al. 1995).

In Sect. 2, we review the data and catalogs, and we describe the homogenization process of these catalogs in Sect. 3. In Sects. 4 and 5, we compute the LFs for bright and faint galaxies. Section 6 is dedicated to the nature of the faintest Coma cluster objects. In Sect. 7, we describe the LF characteristics. Section 8 describes the RS in the Coma CMR. We discuss the inferences of the Coma LF and CMR RS on the cluster building up scenario in Sects. 9 and 10. Finally, Sect. 11 is the conclusion. We assume a distance of $95 \mathrm{Mpc}$ to Coma $\left(H_{0}=75 \mathrm{~km} \mathrm{~s}^{-1} \mathrm{Mpc}^{-1}\right)$. The distance modulus is 34.89 , and therefore the scale is $0.46 \mathrm{kpc} \operatorname{arcsec}^{-1}$. We give magnitudes in the Vega system except if specified otherwise.

\section{Raw data and catalogs}

\subsection{Data}

Here we use the Coma data described in Adami et al. (2006a: A06a hereafter) and already used in Adami et al. (2005a, 2006b). These data were obtained at CFHT with the CFH12K camera using Johnson-like filters in four bands ( $B V R I)$, and reach $R \sim 24$ in an area of $\sim 0.5 \mathrm{deg}^{2}$. We refer the reader to A06a for more details.

To have a homogeneous set of empty fields, we used the VVDS CFH12K imaging fields F02 and F10 (other VVDS CFH12K imaging fields do not have the same wavelength coverage). These two fields were observed with the same $B, V, R$, and $I$ filters (see MC03 for a full description of the F02 field) and have been checked to be free from rich nearby clusters (using VVDS preliminary spectroscopic data). We only used areas where the four bands were simultaneously available. As described later, we completely re-extracted the catalogs with the same parameters from these images, in the most homogeneous possible way compared to Coma data.

A crucial point is that a non-negligible uncertainty source in the LF computation comes from the cosmic variance between

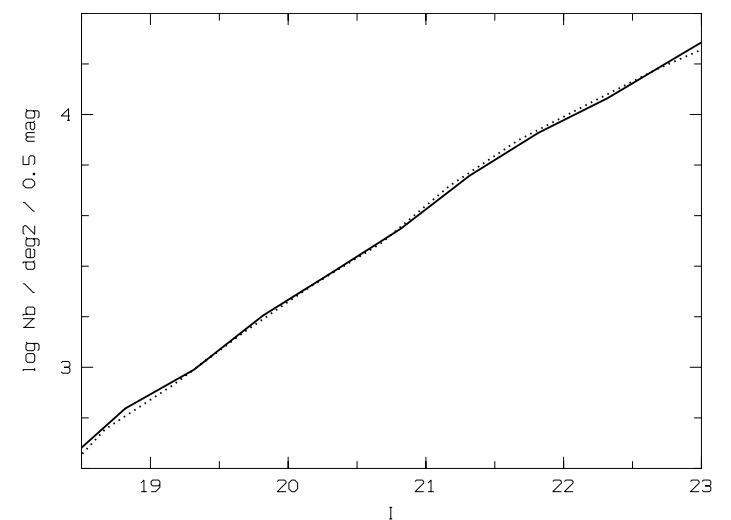

Fig. 1. Differential galaxy counts in the F02 field. Continuous line: original MC03 catalog. Dotted line: our own extraction. The $I$ magnitude range is limited to our mean completeness level: $I=23$.

the Coma line of sight and the comparison fields and inside the comparison fields themselves. As shown later, the total area of our comparison fields is almost equivalent to a $45 \times 45 \mathrm{Mpc}^{2}$ area at $z \sim 0.7$ (the mean F02 sample redshift, Le Fèvre et al. 2005). This is close to the mean size of large-scale structures (e.g., Hoyle \& Vogeley 2004).

\subsection{Object extraction}

Besides having been observed with the same telescope, instrument, and filters, all the data used here were obtained in similar seeing conditions (ranging from 0.8 to 1.07 arcsec, see A06a and MC03). However, the catalogs presented in MC03 have been extracted using much less stringent SExtractor (Bertin \& Arnouts 1996) parameters than in our Coma cluster catalog, giving a quite different completeness. So we decided to re-extract our own catalogs in the Vega system from the F02 and F10 images with exactly the same SExtractor parameters as in A06a. These are mainly a detection threshold of $2 \sigma$ and a minimum number of contiguous pixels above the detection threshold of 9 .

We check that our counts, up to our completeness limit, are in good agreement with those of MC03 for the F02 field in the $I$ band. Corrected by a shift of 0.433 to be translated into the Vega system, we found the MC03 I-band magnitudes to be 0.08 mag brighter than ours (matched to the synthetic star locus, cf. Sect. 3.1). This does not mean that the MC03 I-band magnitudes are wrong by this amount, but that there is a shift of 0.08 mag between the MC03 I-band magnitudes and the spectral templates we used (which are probably not perfect). Applying this shift and comparing the two galaxy counts, we see in Fig. 1 that the agreement is very good.

Finally, since the Coma and the two empty fields have different galactic extinctions, we make a correction using the Schlegel et al. (1998) maps. For the F02 field, we applied a mean magnitude correction on all galaxies because the extinction was only weakly varying across the field with an uncertainty of less than $\sim 0.01$ in the four bands. For the F10 field, we had to locally correct each galaxy using an extinction map with a spatial resolution of 30 arcsec.

\subsection{Star-galaxy separation}

Since the three fields are at different galactic latitudes, we need to remove stars in a homogeneous way in all the fields. We therefore performed the same star-galaxy separation on the three 


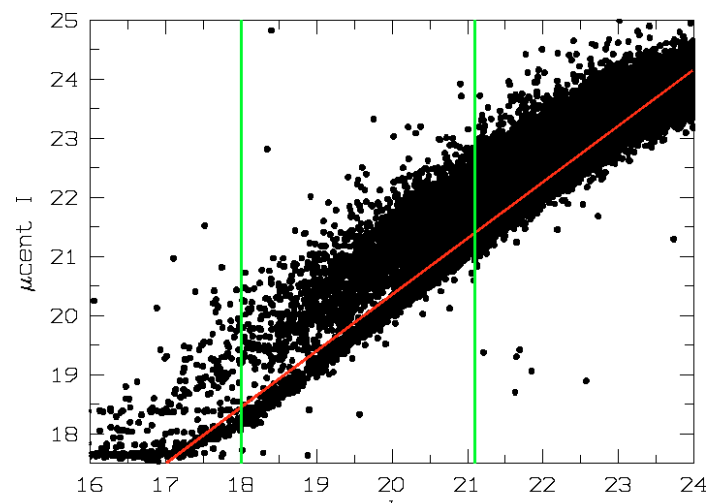

Fig. 2. Star-galaxy separation based on the central I surface brightness (in mag/ $\operatorname{arcsec}^{2}$ ) versus total $I$ magnitude. The thick (red) solid line indicates the separation we applied (in the magnitude range delimited by the two thin (green) vertical continuous lines) between stars and galaxies for one of the F02 subfields.

fields, distinct from the method used by MC03. Our own separation was based on the comparison of total and central $I$-band magnitudes. We started by dividing each of the three fields (Coma, F02, and F10) into subfields of $\sim 0.3 \mathrm{deg}^{2}$ (two subfields for Coma, four for F02, and six for F10) to deal with possible PSF variations across the fields that could have an effect on the star-galaxy separation. We show an example of the star-galaxy separation in one of the F02 subfields in Fig. 2, and we refer the reader to A06a for a full description of the Coma star-galaxy separation and of the general method. We did not use one of the F10 subfields that did not allow an efficient star-galaxy separation because of various star-sequences appearing in the total vs. central I-band magnitude plots, probably because of a strongly varying PSF.

Our star-galaxy separation could probably misclassify extremely compact galaxies in the magnitude range where our separation is efficient ( $I$ brighter than 21). However, in A06a (Fig. 15), we showed that our star counts match star counts from the Besançon model of our Galaxy (Gazelle et al. 1995) very well. The difference between the two counts is $4 \pm 11 \%$ in each magnitude bin. The number of misclassified extremely compact bright galaxies is therefore not a serious concern.

\subsection{Saturated objects and field masking}

As shown in A06a, a significant number of galaxies brighter than $I \sim 18$ are saturated in the Coma field. The F02 and F10 fields are also subject to the same saturation effects and we chose to use the statistical comparison between the Coma and empty fields only for magnitudes fainter than this value. For brighter magnitudes, the LF will be derived with another method described later.

Similarly, very bright objects have extended halos that significantly lower object detection and increase the magnitude error in the concerned regions. We therefore chose to exclude from all fields the areas located at less than twice the radius of objects brighter than $I=18$. Some regions showing low signal-to-noise or diffraction spikes were also excluded manually from the sample. Figure 3 shows the position of all the galaxies in the Coma and F02 fields, the rejected regions, and the splitting into subfields chosen to perform the analysis.

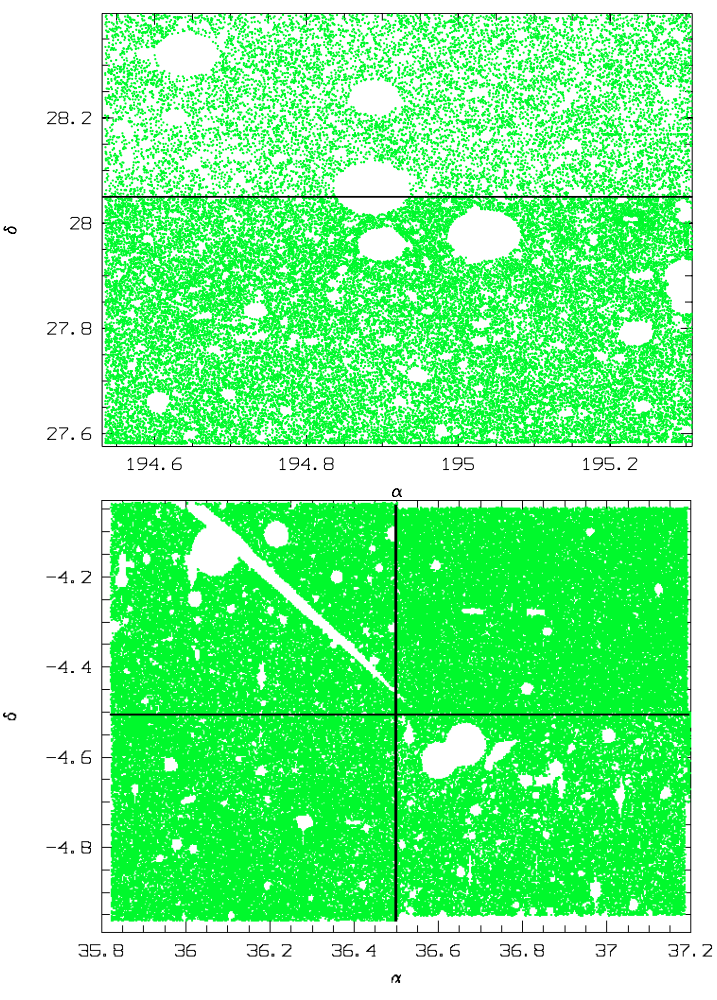

Fig. 3. Top: map of detected objects along the Coma cluster line of sight, showing rejected zones in white. The solid line delineates the two Coma subfields. Bottom: same for the F02 field and subfields. $\alpha$ and $\delta$ are given in decimal degrees, $\alpha$ increasing to the right. We clearly see the shallower depth of the Coma north data in the upper figure.

Table 1. Area coverage for each of the comparison subfields.

\begin{tabular}{cll}
\hline \hline Subfield & Useful area $\left(\mathrm{deg}^{2}\right)$ & Masked percentage \\
\hline F02 subfield 1 & 0.3087 & $17 \%$ \\
F02 subfield 2 & 0.3153 & $15 \%$ \\
F02 subfield 3 & 0.2958 & $21 \%$ \\
F02 subfield 4 & 0.3049 & $18 \%$ \\
F10 subfield 1 & 0.2190 & $21 \%$ \\
F10 subfield 2 & 0.5828 & $37 \%$ \\
F10 subfield 3 & 0.3016 & $30 \%$ \\
F10 subfield 4 & 0.3715 & $10 \%$ \\
F10 subfield 5 & 0.1340 & $4 \%$ \\
\hline
\end{tabular}

\subsection{Area coverage}

We finally obtained a coverage of $\sim 1.2 \mathrm{deg}^{2}, \sim 1.6 \mathrm{deg}^{2}$, and $\sim 0.5 \mathrm{deg}^{2}$ for the F02, F10, and Coma fields, respectively. We note that a small part of the Coma field (about $200 \operatorname{arcmin}^{2}$ at the southwest cluster) was only available in the $R$ - and $I$-bands. We summarize the area coverages in each band for each of the fields and subfields in Table 1.

The total physical area of our comparison fields is about $45 \times 45 \mathrm{Mpc}$ at $z \sim 0.7$. This is of the same order as the periodicity of the Universe large-scale structures (e.g., Hoyle \& Vogeley 2004) and ensures that we will not subtract field counts only coming from a peculiar under- or over-dense field area. Table 2 presents a comparison between this survey and previously available data from the literature in different bands, in terms of photometric depth and surveyed area (both in Coma and in the comparison fields). We quote our point source $90 \%$ completeness levels in this table. The completeness levels of literature data are those quoted in the corresponding papers. We also give 
Table 2. Comparison with recent Coma cluster data from the literature.

\begin{tabular}{cllll}
\hline \hline Filter & $\begin{array}{l}\text { Observed } \\
\text { surface } \\
\left(\mathrm{deg}^{2}\right)\end{array}$ & $\begin{array}{l}\text { Limiting } \\
\text { magnitude }\end{array}$ & $\begin{array}{l}\text { Control } \\
\text { field } \\
\left(\mathrm{deg}^{2}\right)\end{array}$ & Reference \\
\hline$B$ & 0.45 & 24.75 & 2.8 & present survey \\
& 0.20 & 22.5 & 0.17 & AC02 \\
& 5.2 & 21.7 & 0.556 & Bj02 \\
$V$ & 0.187 & 24.0 & 0.037 & T98 \\
& 0.45 & 24.0 & 2.8 & present survey \\
& 0.29 & 23.75 & 0.18 & AC02 \\
$R$ & 0.417 & 22.0 & 0.4 & L97 \\
& 0.50 & 24.0 & 2.8 & present survey \\
& 0.29 & 23.25 & 0.18 & AC02 \\
& 5.2 & 21.7 & 0.278 & Bj02 \\
& 0.0145 & 25.5 & 0.075 & B95 \\
& 0.97 & 20.5 & SDSS & IP03 \\
$I$ & 0.187 & 23.5 & 0.052 & T98 \\
\hline
\end{tabular}

Notes to Table 2: $\mathrm{AC} 02=$ Andreon \& Cuillandre (2002), Bj02 = Beijersbergen et al. (2002), B95 = Bernstein et al. (1995), IP03 = Iglesias-Páramo et al. (2003), L97 = Lobo et al. (1997), T98 = Trentham (1998).

the Coma cluster areas covered, together with the surface of the comparison fields in each case. This clearly shows that among all studies, our survey is the best compromise between depth, coverage, and robustness in the field comparison.

\section{Catalog homogenization}

\subsection{Magnitudes}

Computing LFs based on statistical empty field comparisons requires subtracting the empty field galaxy counts from the Coma cluster galaxy counts. As these counts have very steep slopes at faint magnitudes, even small systematic magnitude uncertainties between the fields can induce significant errors in the final results (e.g., Lobo et al. 1997). We therefore require relative uncertainties between the different fields smaller than 0.05 mag in each photometric band; this upper limit leads to differences of the order of $40 \%$ in our LF that are already prohibitive.

Using the field and Coma catalogs as they are does not generally allow us to reach such a precision because of possible small zero point variations or PSF inhomogeneities. Even relatively modest seeing variations of $\sim 10 \%$ can induce differences of several tenths of magnitudes for faint objects (e.g., Savine 2002), too large for our requirements. We therefore decided to rescale all bands to match the spectrophotometric star template loci in color-color plots. This does not mean that we get the true magnitudes, but that magnitudes in the different fields will be homogenized with a precision of the order of a few $0.01 \mathrm{mag}$.

In each of the different subfields, we selected stars (as defined in A06a) and compared the observed and template star loci in all the possible color-color plots (using $B-, V-, R$-, and $I$-bands). Templates were taken from the library of Pickles (1998). Results after the rescaling are shown in Fig. 4 for one of the subsamples of the F02 field and for one color combination. The values of the individual magnitude shifts applied in each subfield are $0.01 \pm 0.04$ mag for each band, and are smaller than the 0.05 mag limit. Even if they are low, showing that our fields were already quite homogeneous, the effects of these corrections are not negligible on the LF errors. We finally note that the corrections applied to galaxies were computed from star loci. Even though the surface brightness profiles of bright galaxies are different from those of stars, synthetic color-color sequences for

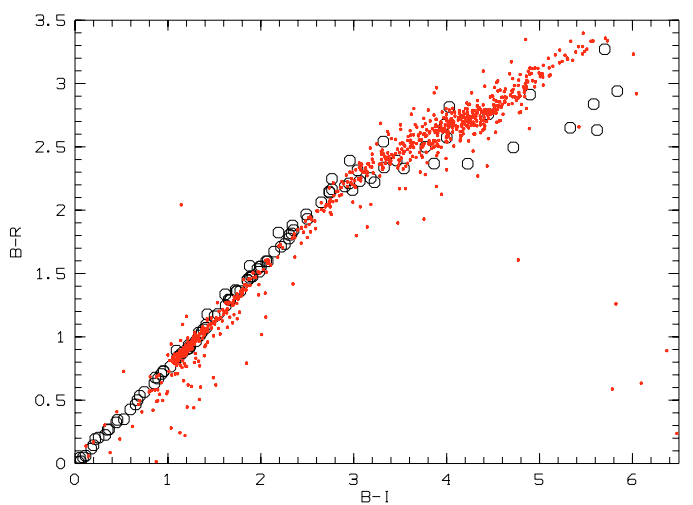

Fig. 4. Color-color plots for one of the F02 subfields after applying the small magnitude shifts described in Sect. 3.1. Small dots are the stars extracted from the F02 observations and circles are the star synthethic models.

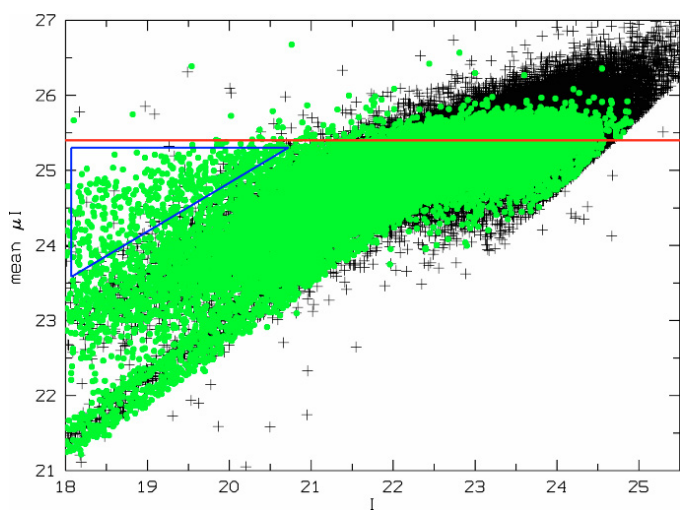

Fig. 5. Mean $I$ surface brightness (in mag/arcsec ${ }^{2}$ ) versus total I magnitude. Crosses are objects from the F02 field and light (green) dots are from the Coma cluster line of sight. The horizontal line indicates the surface brightness cut we applied. The blue triangle indicates the place where low surface brightness objects are in excess in the cluster compared to the field.

stars can still be used advantageously as secondary photometric standards. Indeed, our study is mainly focused on faint galaxies that are at least partially seeing dominated, as stars are.

\subsection{Surface brightness limitations}

As shown in Fig. 5, the F02 and F10 data are deeper than our Coma data in terms of total magnitude and surface brightness. Therefore, to avoid oversubstracting background objects to the Coma counts, we limited both the Coma and the F02 and F10 catalogs to a given surface brightness $(I=25.2, R=26.0$, $V=26.4$, and $B=26.8 \mathrm{mag} \operatorname{arcsec}^{-2}$ ). These values were chosen to maximize the differences between the Coma and empty field counts as a function of magnitude. Figure. 5 illustrates this case in the $I$-band. It is also interesting to note that an excess of low surface brightness galaxies appears in the Coma field, at $I$ lower than 21.5 , a peculiar class of objects already detected in Adami et al. (2006b).

\subsection{Total magnitude completeness limits}

Completeness is not straightforward to estimate and is a complex function of galaxy characteristics and of detection parameters. For example, relatively compact galaxies have a concentrated brightness profile and are easier to detect than low surface 
brightness galaxies. Using simulations, we defined two completeness limits in each of the observed bands in A06a: the $90 \%$ point-source completeness and the $90 \%$ extreme low surface brightness galaxy completeness. These two estimates give the interval where the completeness level should lie for a given class of galaxies. For example, we show that a $90 \%$ completeness level is reached between $R=20.75$ and $R=24$ in the $R$-band (north data) and between $I=20.25$ and $I=23.25$ in the $I$-band (south data) (see A06a). A second way to estimate the mean completeness over all classes is to compare our catalog with deeper catalogs. Using the Bernstein et al. (1995) $R$-band catalog (hereafter B95), we found a mean $R$-band $90 \%$ completeness level of $R \sim 23.5$ (cf. A06a). We present a third method here close to that described in Andreon \& Cuillandre (2002) and using only the catalog data. This method will allow us to give an estimate of the mean completeness level in all bands, resulting from the mean object profile over the whole cluster galaxy population. We proceed as follows:

- First we use the total magnitude range where our data are deep enough to detect all galaxies, whatever their surface brightness. We then consider the upper central surface brightness envelope of the galaxy population as a function of total magnitude, which is only related to the physical constraints between maximum surface brightness and total magnitude. This limit is represented by inclined lines in Fig. 6.

- Second, we define our surface brightness detection limit (horizontal lines in Fig. 6), which is directly linked to our survey characteristics.

The intersection between these two lines gives a conservative estimate of the completeness (method illustrated in Fig. 6). This method gives completeness levels of $I \sim 23$ and $R \sim 23.5$. The $R$ value is very similar to that given by the comparison with the B95 catalog. The $R$ and $I$ values are brighter by 0.5 mag than computed with point source simulations (A06a). We therefore decided to assume a mean completeness 0.5 mag brighter than the point source $90 \%$ completeness levels (given in A06a and that are different between north and south data), whatever the band, namely, $B=24.25, V=23.5, R=23.5$, and $I=23$ for the mean Coma cluster line of sight galaxy population.

It can be noted that the southern field is deeper than the northern field in most of the bands (see A06a), as extensively discussed in A06a. We stress, however, that in the present paper we use the brightest magnitude limit of the north AND south fields to define the completeness levels in each magnitude band. This ensures that no artificial effects will pollute our results: in the magnitude limits we fix, our data are complete whatever the location in the cluster.

\section{Bright galaxy luminosity functions}

We will now describe how we can compute the bright galaxy LF and how it compares to numerical simulations. Computing a LF using redshifts is the most accurate method and must be used as much as possible, but of course it is difficult to achieve redshift completeness down to faint magnitudes.

\subsection{Spectroscopic luminosity functions}

As described in A06a or Adami et al. (2005b), we have compiled the largest sample of spectroscopic redshifts presently available along the Coma cluster line of sight ( $~ 500$ redshifts in the area
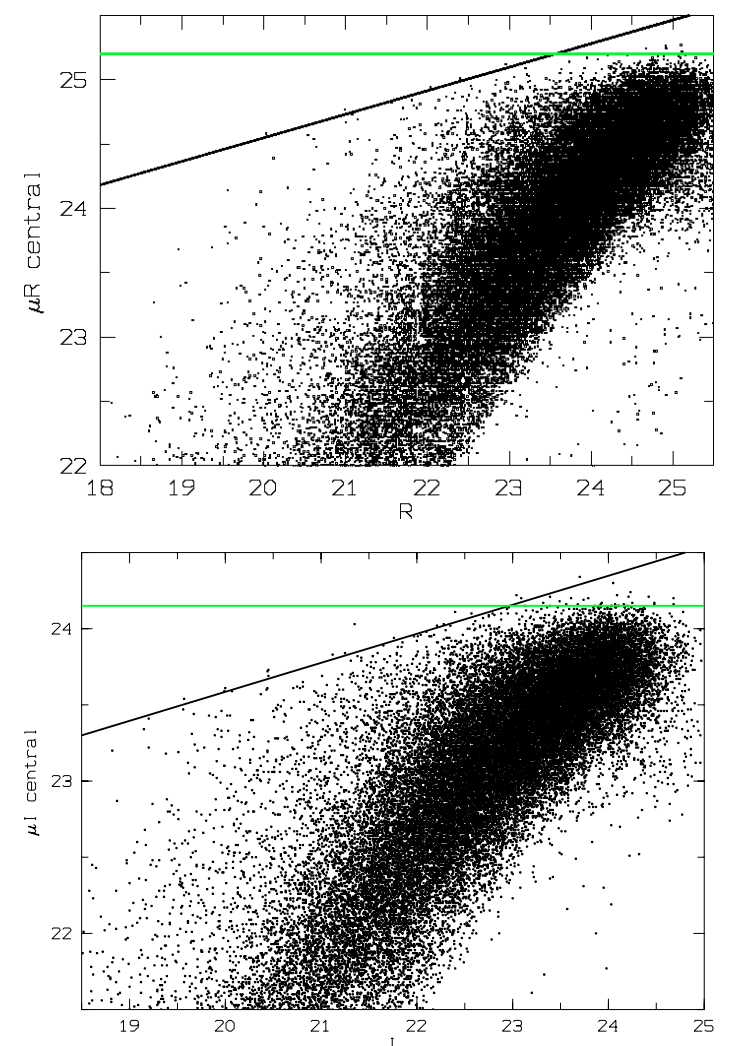

Fig. 6. Central surface brightness (in mag/arcsec ${ }^{2}$ ) versus total magnitude. The thick inclined line gives the intrinsic maximum central surface brightness for a given total magnitude. The horizontal line gives the maximum central surface brightness detectable in our Coma data. The intersection between the two lines gives an estimate of the mean completeness level. Top: $R$-band, bottom: I-band.

covered by our $\mathrm{CFH} 12 \mathrm{~K}$ data). However, we are not $100 \%$ complete in spectroscopy even at bright magnitudes. We therefore applied statistical arguments by computing the percentage of galaxies with a redshift inside Coma for a given magnitude, and applying this percentage to the total number of galaxies (with and without a redshift) inside the bin. We also limited our sample to a $\sim 50 \%$ completeness level. Translated in magnitudes, this gives the following faint ends for the spectroscopic LFs: $I=18.25, R=18.75, V=18.75$, and $B=19.25$. Uncertainties on these LFs due to incompleteness are then simple to compute, since the minimal number of galaxies inside Coma is the number of galaxies with a known redshift inside Coma and the maximum number is the total number of galaxies inside the magnitude bin. This range gives an estimate of the total error (100\%), close to a $3 \sigma$ error. Bright LFs are shown in Fig. 7 for the $B$ and $V$-bands together with their $3 \sigma$ uncertainties, which mainly reflect the redshift catalog incompleteness. LFs are computed in a 0.5 mag running window with a step of $0.25 \mathrm{mag}$.

\subsection{Comparison with simulations}

To compare our bright LF with modeled clusters, we used the simulations described in Lanzoni et al. (2005). Their mass resolution is $10^{9} h^{-1} M_{\odot}$, corresponding to an absolute $B$ magnitude of $\sim-17$ ( $B \sim 18$ at the Coma cluster redshift). This is not sufficient to produce faint galaxies, but a comparison with real data will allow us to validate the simulation process and check that observations and simulations are compatible. 

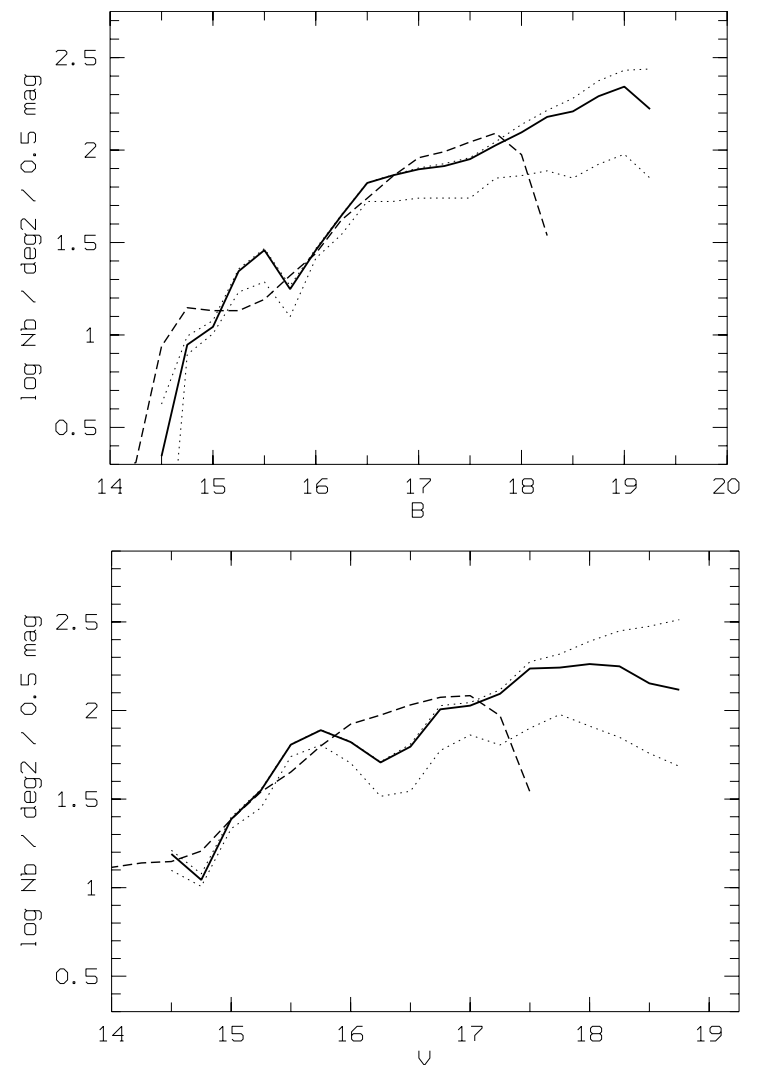

Fig. 7. Coma cluster galaxy LFs computed with spectroscopic redshifts (continuous line) along with the total error envelope (dotted lines). From top to bottom: $B$ and $V$. Simulated LFs are shown as a dashed line.

We extracted a LF from the simulations inside a circle of 0.3 virial radius, the same physical area as our Coma cluster data. We used a $r_{200}$ virial radius of $1.5 h^{-1} \mathrm{Mpc}$ (Geller et al. 1999) for the Coma cluster. We renormalized the number of galaxies by the ratio between the Coma cluster mass in the considered area and the total mass of the systems simulated by Lanzoni et al. (2005), based on the 15 simulated clusters with masses ranging between $10^{14}$ and $10^{15} M_{\odot}$. The result of the comparison is shown in Fig. 7 in the $B$ - and $V$-bands. The agreement appears very good, except for the facts that:

- the LF appears smoother in the simulated clusters than in Coma;

- the simulated LF does not follow the observations above $V \sim 17$ and $B \sim 18$ because of the lack of mass resolution. The dips seen in the Coma cluster LFs (significant given the $3 \sigma$ uncertainties) are probably due to peculiar processes in the Coma cluster not reproduced by generic cosmological simulations; we will discuss that later.

\section{Faint galaxy luminosity functions}

To select the objects in Coma beyond the available spectroscopic data, we use two methods described in this section along with the resulting uncertainty estimates.

\subsection{Faint luminosity function using simple statistical subtractions}

In this first approach, the number of galaxies is expressed along the Coma cluster line of sight as a function of magnitude, whatever their colors:

$N_{\mathrm{C} \text { los }}(\mathrm{mag})=N_{\mathrm{C}}(\mathrm{mag})+N_{\text {efield C }}(\mathrm{mag})$,

with $N_{\mathrm{C}}$ los the number of objects along the Coma line of sight, $N_{\mathrm{C}}$ the number of objects inside the Coma cluster, and $N_{\text {efield C }}$ the number of background and foreground objects at the Coma position (empty field). From the F02 and F10 fields, we obtained an estimate of $N_{\text {efield }}$ (mag) in the field, but at positions different from the Coma cluster. If our comparison fields are large enough to smooth the field-to-field variance, then $N_{\text {efield }}$ (mag) $N_{\text {efield C }}(\mathrm{mag})$ should have a null mean and we get:

$N_{\mathrm{C}}(\mathrm{mag})=N_{\mathrm{C} \text { los }}(\mathrm{mag})-N_{\text {efield }}(\mathrm{mag})$,

with an uncertainty that will be estimated in the following. LFs are computed in a 0.5 mag running window with a step of $0.25 \mathrm{mag}$.

\subsection{Faint luminosity function using color-dependent statistical subtractions}

This time, we use the same method, considering the galaxy counts not only as a function of magnitude, but also as a function of colors, to allow for example selections on colors and estimates of LFs for different classes of galaxies. The estimate of the Coma cluster counts then becomes:

$N_{\mathrm{C}}\left(\mathrm{mag}, \operatorname{col}_{i}\right)=N_{\mathrm{C} \text { los }}\left(\operatorname{mag}, \operatorname{col}_{i}\right)-N_{\text {efield }}\left(\operatorname{mag}, \operatorname{col}_{i}\right)$,

with $\mathrm{col}_{i}$ being all colors available. $N_{\mathrm{C}}(\mathrm{mag})$ is then the sum of $N_{\mathrm{C}}\left(\mathrm{mag}\right.$, color $\left._{i}\right)$ over all color ${ }_{i}$ in the galaxy sample.

A good way to understand this type of method is to consider only one color. In this case, the method basically consists of estimating the difference between the Coma cluster line of sight and the empty field F02 and F10 CMRs. As an illustration, we will describe the computation of the $B-R / R \mathrm{CMR}$ and the detection of a RS inside the Coma cluster down to very faint magnitudes in the following.

\subsection{Luminosity function uncertainties}

Uncertainties on the counts are of several origins. The first uncertainty is the Poissonian error simply related to the limited number of galaxies in each magnitude bin in the empty fields or in the Coma field. Other sources of errors are related to the magnitude accuracy and to the distribution of galaxies in the different fields. Magnitude uncertainties lead to errors on galaxy counts that propagate into LF uncertainties when computing the difference between the Coma line of sight and the empty field counts. These uncertainties can be estimated as a function of magnitude with Monte-Carlo simulations by generating catalogs of objects with magnitudes Gaussianly distributed according to the data statistics given in A06a, and computing 100 LFs. The whole sample of generated LFs therefore allows us to estimate the uncertainty as a function of magnitude.

Another source of uncertainties on the background counts and LFs is the field-to-field variance, and a way to deal with this effect is to use many comparison fields and get the fieldto-field variance from the fluctuations on the measured counts. This method was used for example by Bernstein et al. (1995), but 
Table 3. Parameters used for empty field luminosity and correlation functions.

\begin{tabular}{lllll}
\hline \hline Filter & $B$ & $V$ & $R$ & $I$ \\
\hline Schechter $\alpha$ & -1.23 & -1.39 & -1.44 & -1.45 \\
Schechter Vega $\mathrm{M}^{*}$ & -21.0 & -22.0 & -22.7 & -23.3 \\
Power law $\gamma$ & 1.596 & 1.548 & 1.612 & 1.628 \\
Power law $\mathrm{r}_{0}$ & 2.485 & 2.545 & 2.575 & 2.725 \\
\hline
\end{tabular}

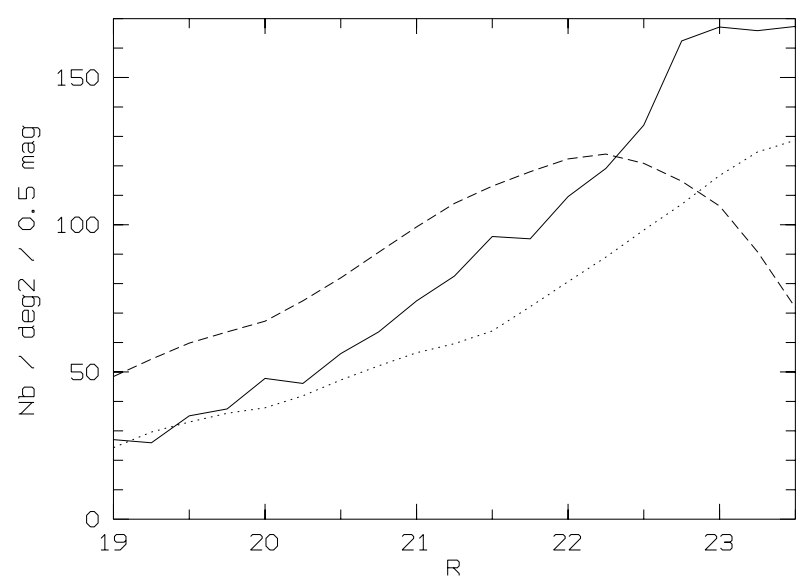

Fig. 8. Three uncertainty sources for the zone of Coma in the $R$-band: dashed line: error from the field-to-field variance, solid line: error from magnitude uncertainties, and dotted line: Poisson error.

as we do not have enough independent comparison fields (only two), we use a method applicable on a single field and described by Huang et al. (1997). Andreon \& Cuillandre (2002) have underlined the fact that relatively strong variations of the luminosity and correlation functions induce variations of only a few percent on the errors induced by the cosmic variance. However, we have decided to estimate this error. Following Huang et al. (1997), we computed the correlation function in the F02 field using the VVDS redshifts with the selection criteria used for our photometric samples. We then assumed the correlation function of the F10 and of the Coma line of sight to be the same. Regarding LFs, we used the Ilbert et al. (2005) study to estimate a mean value of the Schechter parametrisation within our selection criteria. The parameters we used are given in Table 3.

Finally, all these errors were quadratically added to obtain the error budget for the faint parts of the LF. Figure 8 shows the three contributions to the LF uncertainty in the Coma area in the $R$-band. At bright magnitudes, the field-to-field variance error dominates, while the error due to the uncertainty on magnitudes is the largest one at faint magnitudes. Given the relatively low level of uncertainty due to field-to-field variance, the counts of galaxies between the F02 and F10 fields should be very similar. Between $R=19$ and 23.5, the difference between the numbers of galaxies in the F02 and F10 fields in 0.25 mag bins is indeed very small: $-71 \pm 120$ galaxies $/ \mathrm{deg}^{2} / 0.25 \mathrm{mag}$; this represents about $2 \%$ of the total number of galaxies inside the considered bins.

\section{Expected contamination by globular clusters at the faint end of the LF}

The faintest objects found to be in the Coma cluster by the statistical subtraction method can be either galaxies or globular clusters (GC hereafter). Using a rather small area, Bernstein et al. (1995) have shown that the GC contribution becomes
Table 4. Percentage of total number of objects with colors similar to GCs for the Coma subfields (see Figs. 9-12). Typical $1 \sigma$ errors on the total number counts are also given.

\begin{tabular}{llllllll}
\hline \hline Subfield & 4 & 5 & 8 & 9 & 10 & 13 & 14 \\
\hline$\%$ & $25 \%$ & $37 \%$ & $66 \%$ & $37 \%$ & $35 \%$ & $8 \%$ & $30 \%$ \\
Error & $21 \%$ & $20 \%$ & $21 \%$ & $21 \%$ & $20 \%$ & $18 \%$ & $22 \%$ \\
\hline Subfield & 15 & 18 & 19 & 20 & & & \\
\hline$\%$ & $52 \%$ & $14 \%$ & $32 \%$ & $36 \%$ & & & \\
Error & $20 \%$ & $20 \%$ & $20 \%$ & $21 \%$ & & & \\
\hline
\end{tabular}

significant for $R$ fainter than 23.5. However, Marín-Franch \& Aparicio (2003) reach apparently contradictory conclusions. Therefore, to estimate the relative fractions of these objects in our data, we used the description of the GC population around NGC 4406 given by Rhode \& Zepf (2004). This galaxy (also known as M86) is an elliptical cluster galaxy attached to the Virgo cluster. Its GC population is therefore typical of the GCs we could detect in Coma. According to Rhode \& Zepf (2004), GCs extend to less than twice the typical galaxy optical radius from the NGC 4406 center, and given our masking conventions near bright galaxies, the GCs still attached to galaxies brighter than $I=18$ are not counted in our LFs. The only GCs counted are those attached to fainter galaxies (probably a minor contribution) and those expelled from their parent galaxies that we will discuss now.

The brightest GC in NGC 4406 has a magnitude of $V \sim 20$, which, translated to the Coma cluster distance (assuming a Virgo distance of $16.7 \mathrm{Mpc}$ ), gives a $V$ magnitude of $\sim 23.75$. This means that the population of single GCs detected in our data is probably negligible at $V$ brighter than 23.75 , and we will only consider the fainter magnitude bins as potentially polluted by GCs.

To estimate the number of GCs in the next $\sim$ half magnitude bin, we used the color-color plot of Rhode \& Zepf (2004), which gives the location of their candidate GCs in the $B-V$ versus $V-R$ plane. Their $B$ and $V$ filters are very close to our own filters (Johnson-like filters), while their $R$ filter is close to a Cousins filter, and we correct their $R$ values by -0.1 mag assuming that GCs have elliptical-like colors (Fukugita et al. 1995). With the same assumption, we also shifted the $B-V$ and $V-R$ colors from the Virgo distance to the Coma distance.

We then computed the $B-V$ versus $V-R$ map of objects statistically in the Coma cluster (obtained by subtracting the empty field $B-V$ versus $V-R$ map from the Coma line of sight $B-V$ versus $V-R$ map, after renormalizing them by their respective areas), in which we approximately defined the GC location from Rhode \& Zepf (2004). This allowed us to compute the percentages of objects in these maps located at the same place as GCs. These percentages, given in Table 4, are only upper values of the number of globular clusters as regular faint Coma cluster galaxies can have similar colors. The result is a complex interplay of object colors and, of course, these percentages are only valid in the magnitude ranges in which the completeness in all three $B$-, $V$-, and $R$-bands is reasonable.

Using for example the point source $90 \%$ completeness levels of A06a (applicable to GCs, which are nearly point sources at the distance of Coma), we have complete $V$-band data down to $V=24-24.25$, about half a magnitude deeper than the bright end of the GC LF. $R$-band data are deeper than this for nearly all object types. However, $B$-band data are only complete down $B=24.75$. So, for objects with $B-V$ greater than 0.75 (typically elliptical-like objects), we can estimate the GC percentages 
down $V \sim 24$. For $B-V$ greater than 0.5 (typically spiral-like objects), we can estimate the GC percentages down to $V \sim 24.25$. For these reasons, not all regions provided a GC percentage: we limited our analysis to the regions where more than 15 objects were statistically in Coma, detected both in $B, V$, and $R$ and fainter than $V=23.75$ (the bright end of the GC LF).

For magnitudes fainter than $B=24.75$, we have no way to unambiguously estimate what the GC contamination in our LFs using colors will be. We know, however, that given the GC physical sizes, such objects will be unresolved in our data. Objects significantly larger than the seeing size are therefore likely to be galaxies rather than GCs (unless several GCs are close to each other in order to mimic objects larger than the seeing). If we consider the $R$-band major axis of objects fainter than $B=24.75$, we find that $53 \%$ of these objects are two times larger than the seeing in $R$. This means that a significant part of the very faint object population in Coma ( $B$ fainter than 24.75) is unlikely to be GCs.

Using a bootstrap technique with 500 resamplings, we also estimated the $1 \sigma$ level uncertainty on the total number of objects (see Table 4). If the percentage of GCs is smaller than three times this uncertainty, we considered the GC population as not significant. We clearly see that only subfields 8 and 15 have a potentially significant GC population.

We also independently searched for regions with a significant GC percentage by summing all the densities given by Marín-Franch \& Aparicio (2003). Among our subfields 8, 9, 13, 14,18 , and 19, only subfield 8 has a significant GC population (at a level larger than $3 \sigma$ ): these authors found a GC density of $0.0545 \pm 0.018$ in the vicinity of NGC 4874 . Subfield 8 mainly includes this galaxy and is very close to the field surveyed by Bernstein et al. (1995), who also concluded that there was a significant population of GCs.

\section{Luminosity function characteristics}

We first note that the general shape of the bright part of the galaxy LF is similar over the whole field. Besides the dips discussed later, the faint LF parts (typically $R$ fainter than 19) show spatial variations discussed in this section.

\subsection{Luminosity function across the Coma field}

To sample possible environmental effects on the LF, we cut the Coma field in twenty $10 \times 10 \mathrm{arcmin}^{2}$ areas. Results for the corresponding LFs are shown in Figs. 9-12 for the four available bands (computed with the simple statistical subtraction method). For all photometric bands and individual exposures, there is clearly a dichotomy between the north-northeast regions, with a steeply rising LF, and the south-southwest regions with flatter LFs. This could be due to different galaxy types that behave differently. To address this observation, we used the colordependent statistical subtraction method in two different ways.

In a first approach, we compute LFs for blue and red galaxies selected using the CMR RS defined in A06a. This RS has a given width, and we considered the galaxies statistically in Coma and under the RS at the significance level of $1 \sigma$ as blue. Other galaxies statistically in Coma were considered as red. This separation corresponds at first order to a classification into early- and latetype galaxies (e.g., Fukugita et al. 1995). The results are given in Fig. 13, and we clearly see that the blue object fraction increases toward faint magnitudes. This is in good agreement with the results by Odell et al. (2002). Figure. 13 is, however, not directly comparable to the $R$-band LFs given in Fig. 11 . This is because it uses the color-dependent statistical subtraction method with $B$ and $R$, while Fig. 11 uses the simple statistical subtraction method. The $B$-band data are not as deep as the $R$-band data: a galaxy has to be detected both in $B$ and $R$ to be included in the color LF. Relatively faint and very low star-forming rate objects will be excluded because they are fainter in the $B$-band than in the $R$-band.

As a second approach we use synthetic colors, which provide a robust classification of galaxies into spectrophotometric types. The lack of deep $U$-band data prevents any reliable computation of photometric redshifts in Coma (e.g., Savine 2000), but a color classification of galaxies is still possible using statistical field subtractions in the color-space. Galaxy templates from Coleman et al. (1980) were used to derive the expected colors of galaxies at the redshift of Coma and discriminate between different (extreme) galaxy types in the color-space, from E to Im galaxies. Different simulations were performed to estimate the contamination level expected between adjacent types when including realistic photometric uncertainties. As a result, we define three galaxy populations, separated in the $B-I / B-R$ space by two lines:

$(B-I)=-1.2(B-R)+1.45$

and

$(B-I)=-1.2(B-R)+2.60$.

This classification roughly corresponds to a separation between early-type, early-spiral, and late-spiral galaxies. With these limits, only $15 \%$ of the Coleman et al. (1980) type 2 (Sbc) are misclassified as type $1(\mathrm{E} / \mathrm{S} 0)$ at the typical S/N levels of the faintest galaxies in the sample. Similarly, only $26 \%$ of type $3 / 4$ galaxies (Scd/Irr) are classified as type 2 .

The first and second approaches are complementary. While the CMR only depends on the early-type galaxy observed colors, the synthetic color modeling depends on the templates used. The second method is more predictive (subdivision into three galaxy classes instead of two), but also more model-dependent. The fact that these two methods provide similar results gives us confidence in our results.

Whatever the galaxy colors or types, the LFs in the southsouthwest regions remain flat (see Fig. 14 for the three populations defined in the color-space), except perhaps at the faint end for the early and late spiral galaxies. The situation is more complex in the north-northeast parts of the cluster. Red galaxies have a similar behavior to early-type galaxies. Early spirals clearly dominate late spirals (as expected in a massive cluster), except perhaps in the western parts such as subfield 5, where the numbers of early and late spirals are comparable. The steep LF rise is due to red/early-type galaxies for $R<22.5$ and to blue/spiral galaxies after.

\subsection{Faint Coma cluster luminosity function around the main cluster galaxies}

We now describe the LF shapes, which are quite different, around the main cluster galaxies and groups in the Coma field, i.e., NGC 4874, NGC 4889, and NGC 4911 (see Figs. 9-12). NGC 4911 is a well known galaxy attached to an infalling group (e.g., Neumann et al. 2003), while NGC 4874 and NGC 4889 are the two central galaxies of the cluster. Note that we are not sampling the NGC 4839 region, another infalling group, in our data.

In the NGC 4889 field, LFs are steeply rising in all photometric bands up to $B \sim 23.5, V \sim 23, R \sim 23$, and $I \sim 23$, then 


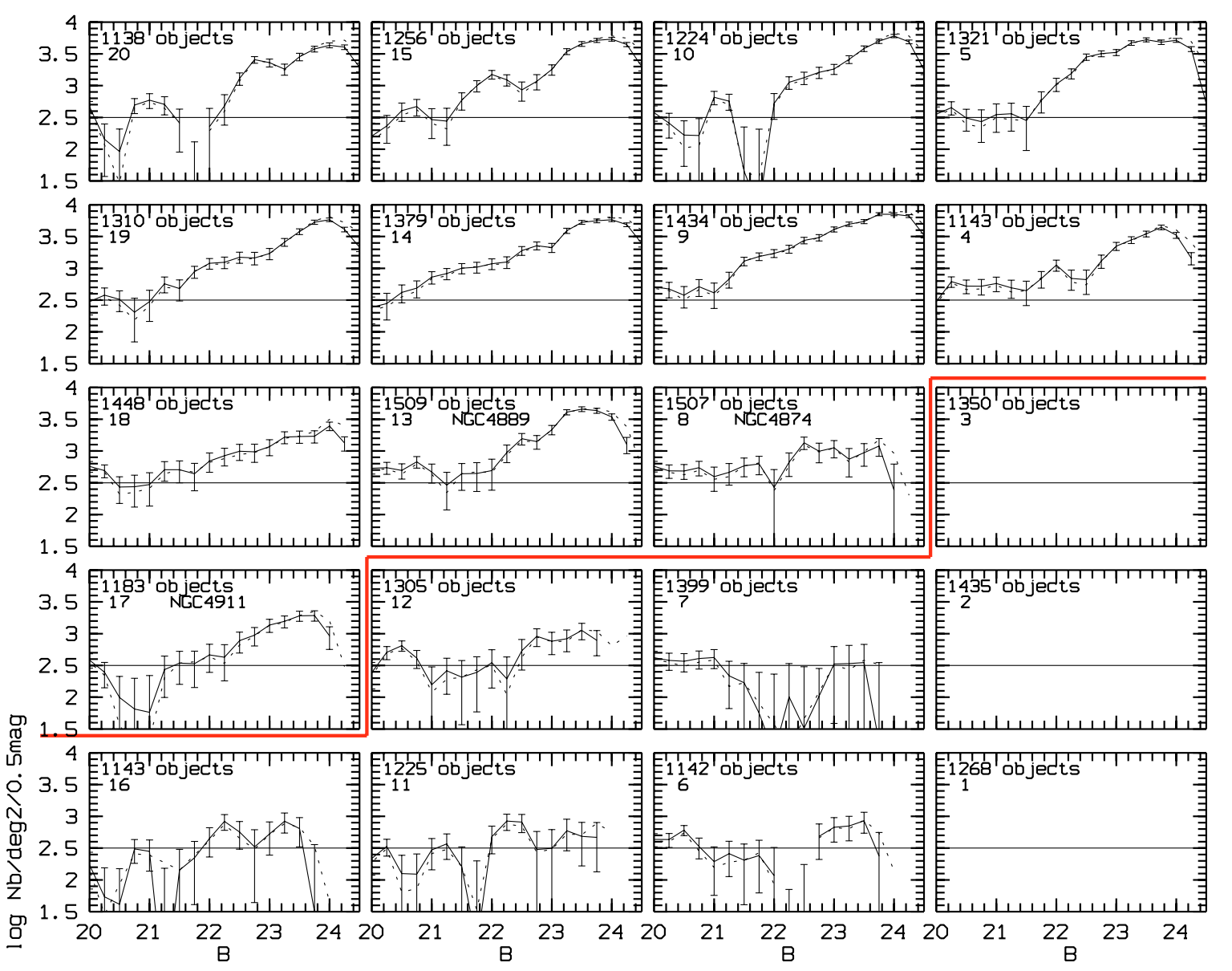

Fig. 9. $B$-band LFs for different locations in the Coma cluster. North is top and east is left. The locations of the three main galaxies (NGC 4874, NGC 4889, and NGC 4911) are also indicated, together with the total number of galaxies and with the number of galaxies brighter than $R=23.5$ inside each line of sight. The empty sub-graphs correspond to areas where we did not have $B$ and $V$ data for technical reasons. The red solid line separates the north-northeast and south-southwest regions. Continuous lines correspond to the F02 comparisons and dotted lines to the F10 comparisons. The horizontal lines in each subgraph are only given as a visual reference to be able to more easily compare results between the different regions.

decrease above these limits in the $B$ - and $V$-bands, while continuously rising or at least staying flat in $R$ and $I$. In the NGC 4874 field, the LF is moderatly rising in $B$ and $V$, with a steeper rising in $R$ and $I$, and a maximum reached around $R \sim 23$ and $I \sim 22.5$. In the field of NGC 4911, the LF is moderately rising in $B$ and $V$ up to $B \sim 23.75$ and $V \sim 23.5$. In $R$ and $I$, the LF is oscillating, but still rising up to $R \sim 23$ and over the whole magnitude range in $I$. We will discuss in the following the link between these shapes and the general cluster building history.

\subsection{Modeling the Coma cluster luminosity function}

We will now give a Schechter modeling of our LFs. This is not a crucial step in our study as we do not use this model, but it can be useful for readers to compare our results with other studies in the literature. We used a single Schechter model of our LFs fitted to the data using an IDL code already discussed in Durret et al. (2002). However, given the clearly non-Schechter LF shapes (with many bumps and dips), the results are only indicative. The less constrained parameter is $M^{*}$, while the faint magnitude slope of the Schechter function is better constrained. This induces degeneracies between the two parameters, but we did not try to perform a finer analysis given the limited goals of this modeling.
As shown in the last subsection, we can divide the Coma cluster in two parts. A north-northeast (fields 20, 19, 18, 17, $15,14,13,10,9,8,5$, and 4) and a south-southwest part (fields $16,12,11,7,6,3,2$, and 1). For simplicity, we will call these fields the north and the south fields respectively. LFs for these two regions as a whole are shown in Figs. 19 and 20, and the modeling results are given in Table 5. North is modeled by a Schechter function with a steep slope due to red/early type galaxies at bright magnitudes, and to blue/late type objects at very faint magnitudes, while on the contrary, the south LF is nearly flat for all objects. The values of $M^{*}$ are very poorly constrained and their values in $B, V$, and $R$ for the north and south regions are consistent within error bars. However, we can note that the south values are always fainter than in the north (the difference is significant at the $3 \sigma$ level for the $I$-band). We will not discuss this point further, as uncertainties are very large and LF shapes are clearly not Schechter-like.

\subsection{Comparison with literature luminosity functions}

We present in this section a short comparison of our Coma LFs derived from the simple statistical subtraction method (without use of color information) with those derived by Bernstein et al. (1995), Lobo et al. (1997), Trentham (1998), Andreon \& Cuillandre (2002), Beijersbergen et al. (2002), and 

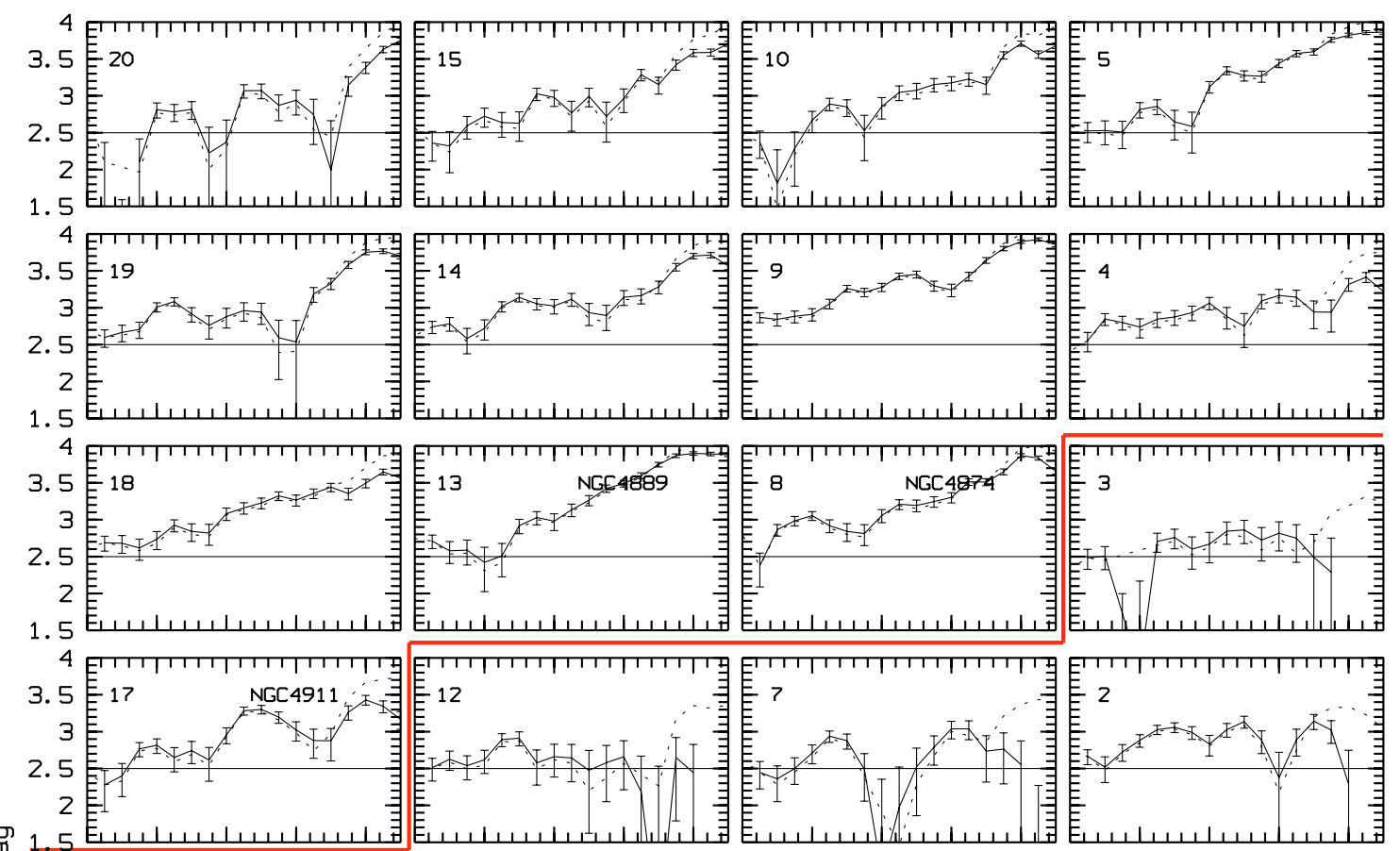

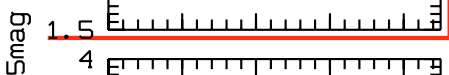
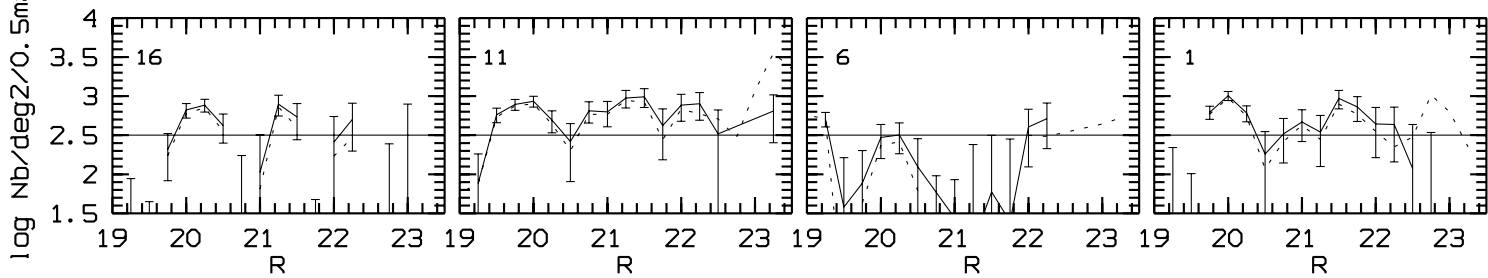

Fig. 11. Same as Fig. 9 for the $R$-band.

Iglesias-Páramo et al. (2003). These authors also use statistical background galaxy subtraction methods, but most of the time in smaller fields of view or with limited depth or less detailed spatial LF analysis. LFs by Bernstein et al. (1995) in the $R$ band, Trentham (1998) in the $B$ - and $R$-bands, and Beijersbergen et al. (2002) are given for areas included in our data, but with an overlap of less than $2 \mathrm{mag}$ in the case of Beijersbergen et al. (2002). We therefore computed our LFs in the corresponding bands only for the Bernstein et al. (1995) and Trentham (1998) fields. Results are shown in Figs. 15 and 16. Given the error bars, the agreement is globaly good in all bands except that the dip (at $R \sim 22$ ) visible in the Bernstein et al. (1995) LF is less prominent in our results. This is probably due to the small comparison area used by these authors. We have only partial overlaps with Lobo et al. (1997), Andreon \& Cuillandre (2002), and Iglesias-Páramo et al. (2003), and only checked that we also have a reasonable agreement with their results. We also have a small magnitude overlap with the recent survey by Milne et al. (2006). They used deep HST images but on a very small area $\left(4.6 \operatorname{arcmin}^{2}\right)$ making difficult a direct comparison with our data. However, fitting a Schechter function in the same area, we find a slope of -1.90 , in relatively good agreement with the Milne et al. (2006) slope of $-2.29 \pm 0.33$.

\section{Red sequences in the Coma cluster}

Using color dependent statistical subtractions naturally leads to investigate the possible existence of a RS at faint magnitudes in the Coma CMR $(B-R$ vs. $R$ ). We will describe here the origin of this RS and the consequences of its characteristics.

\subsection{Why a red sequence in the Coma cluster?}

The existence of a correlation between early-type galaxies and their colors has been known for almost 50 years (e.g., Baum 1959): elliptical and S0 galaxies seem distributed along a RS. This RS proved to be even more obvious in clusters of galaxies, where galaxy evolution is different from that in the field because of environmental effects. Sandage (1972) has shown for example the existence of a very clear RS for the elliptical galaxies of the Virgo and Coma clusters. The use of the RS of early-type galaxies in the CMR rapidly became a common tool to study galaxy clusters (e.g., Mazure et al. 1988; Yee et al. 1999). There is also a visible sequence for late type and dwarf galaxies (Terlevich et al. 2001), but this sequence is more dispersed and shows different colors compared to the elliptical galaxy RS.

The RS in the CMR is mainly due to metallicity effects in galactic systems of various masses (Kodama \& Arimoto 1997). At the Coma redshift, the $B-R$ color is a good tracer of the ratio between the galactic star-forming rate and the galactic mass, and therefore the RS traces the star formation history of these galaxies and is directly related to the metal abundances. The more massive a galaxy, the more easily it will retain metals against dissipative processes such as supernova winds. The more metals present in a galaxy, the reddest the galaxy will be. This is a 

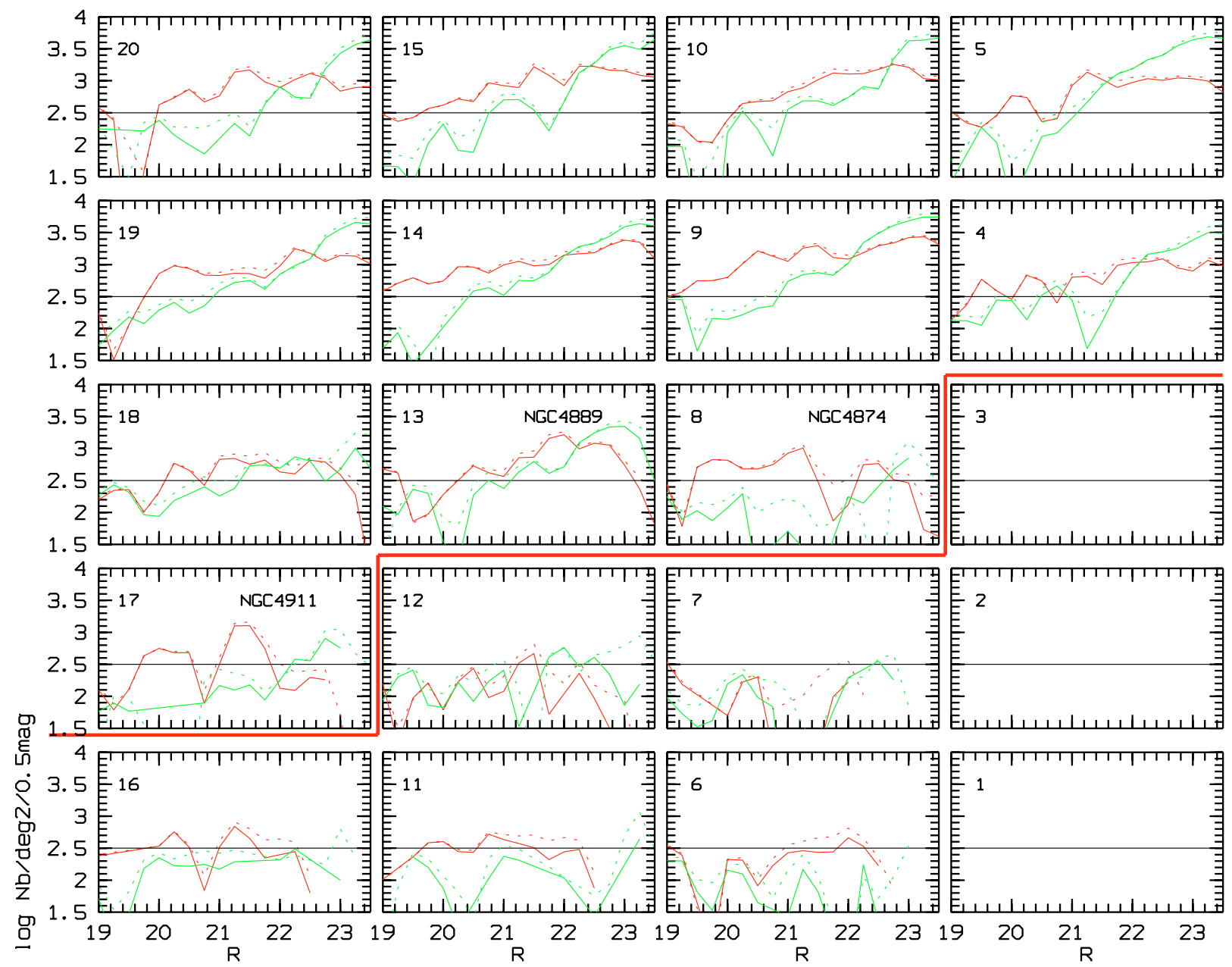

Fig. 13. Same as Fig. 9 for red galaxies (black/red lines) and blue galaxies (grey/green lines). Error bars are not shown for clarity.

simple way to account for the negative slope of the RS in clusters of galaxies.

These characteristics are well established for relatively bright galaxies, but little is known for low-mass galaxies beyond the spectroscopic limit. Authors as Terlevich et al. (2001) already extensively discussed the Coma CMR characteristics, but these previous studies typically end where our sample starts, and the magnitude range beginning at $M_{R} \sim-14$ basically remains an unexplored domain regarding the CMR. Even deep spectroscopic surveys (e.g., Adami et al. 2000 or A06a) were not able to significantly sample the CMR in Coma at magnitudes fainter than $R \sim 21$. However, this range is of major interest because it contains low-mass galaxies with various histories, and for example, we showed in Adami et al. (2006b) that faint low surface brightness galaxies in the Coma cluster are not all located on the $\mathrm{RS}$. We will now investigate the CMR in the Coma cluster down to $M_{R} \sim-11.5$.

\subsection{Removal of field galaxies}

As described earlier, to remove field galaxies, we computed density maps of the Coma line of sight and of the empty field in the $B-R / R$ space. This was achieved via kernel techniques (e.g., Biviano et al. 1996) providing a good compromise between space sampling and resolution of the maps. We then renormalized the two maps to the same area, before subtracting the empty field map to the Coma line of sight map. The resulting map is therefore the $B-R / R$ distribution for objects statistically in Coma (see Fig. 17).

\subsection{Significance of the $B-R / R$ maps}

To interpret the maps given in Fig. 17, we must assess their significance level. The galaxy density $D$ in these $B-R / R$ maps is computed as the difference between the galaxy density along the Coma line of sight $\left(D_{\mathrm{C} \text { los }}\right)$ and along the empty field $\left(D_{\mathrm{E} \mathrm{f}}\right)$ :

$D(B-R, R)=D_{\mathrm{C} \text { los }}(B-R, R)-D_{\mathrm{E}}(B-R, R)$.

We used 500 bootstrap resamplings to compute uncertainties on $D_{\mathrm{C}}$ los and $D_{\mathrm{E} \text { f }}$. The total uncertainty on D is the quadratical sum:

$\sigma_{D}^{2}(B-R, R)=\sigma_{\mathrm{C} \text { los }}^{2}(B-R, R)+\sigma_{\mathrm{E} f}^{2}(B-R, R)$.

We finally compute the significance level maps (Fig. 18) dividing $D(B-R, R)$ by $\sigma_{D}(B-R, R)$. We see that most of the populated regions in Fig. 17 are significant at more than the 3- $\sigma$ level according to Fig. 18. Some regions where there are more objects in the empty fields compared to the Coma line of sight or where the significance level is very low also appear in Fig. 18. They mainly affect some of the Coma south fields in faint and blue areas (and a few bright regions obviously not populated as shown in Fig. 17). 
Table 5. Schechter function parameters for the north-northeast $(\mathrm{N})$ and south-southwest (S) fields.

\begin{tabular}{lrrrr}
\hline \hline & $B$ & $V$ & $R$ & $I$ \\
\hline $\mathrm{N} \alpha$ & $-1.48 \pm 0.03$ & $-1.72 \pm 0.01$ & $-1.74 \pm 0.02$ & $-1.60 \pm 0.01$ \\
$\mathrm{~N} \mathrm{M}^{*}$ & $14.89 \pm 0.46$ & $12.90 \pm 0.24$ & $14.68 \pm 0.91$ & $12.31 \pm 0.23$ \\
$\mathrm{~S} \alpha$ & $-1.32 \pm 0.03$ & $-1.27 \pm 0.03$ & $-1.28 \pm 0.05$ & $-1.27 \pm 0.02$ \\
$\mathrm{~S} \mathrm{M}^{*}$ & $15.86 \pm 0.44$ & $14.82 \pm 0.99$ & $16.05 \pm 0.36$ & $14.29 \pm 0.25$ \\
\hline
\end{tabular}

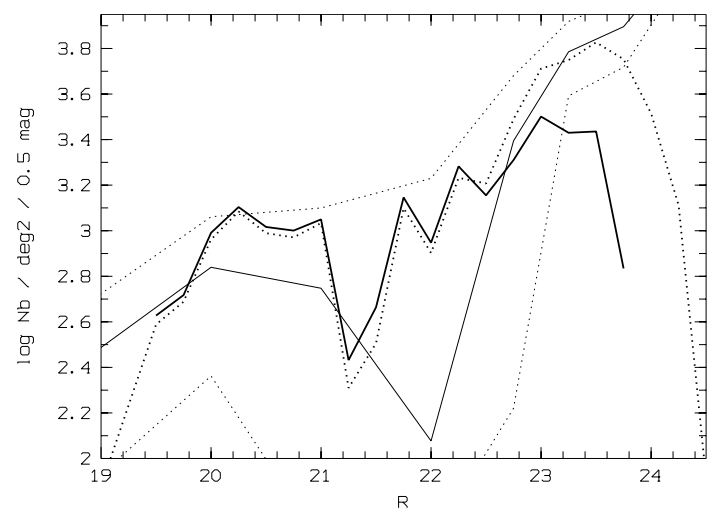

Fig. 15. Comparison with Bernstein et al. (1995: $R$-band). Our own LF estimates are the thick lines, the solid line is the F02 comparison field, and the dotted line for the F10 comparison field. The thin solid line is the literature estimate along with its $1 \sigma$ errors (thin dotted lines).

\subsection{Global distribution in the $B-R / R$ space}

In Fig. 17 we show the areas where we detect galaxies statistically belonging to the Coma cluster, as a function of location in the cluster. For comparison, the RS computed for galaxies brighter than $R \sim 18.5$ in A06a is overplotted on these graphs. Above $R \sim 19.5$, where there is only incomplete or no spectroscopic information, we can clearly see positive and significant galaxy concentrations, most of the time following the bright galaxy RS, whatever the location in the cluster. This is in good agreement with the results of Odell et al. (2002), showing a linear RS from -13 to -22 in a narrow-band filter.

In addition to the RS, extensions towards the red parts of the $B-R / R$ plots show an excess of very red objects (significant and distinct from the ones we will discuss in Sect. 8.4) along the Coma cluster line of sight compared to the field. These objects are so red $(B-R \sim 2.5)$ that they are difficult to explain as part of the Coma cluster itself, because even very red early type galaxies are not so red most of the time (e.g., Fukugita et al. 1995). Only atypical dust contents can provide such red colors at the Coma cluster redshift. Moreover, this contribution is not negligible and objects with $B-R$ greater than 2 account for $19 \%$ of all the objects potentially belonging to the Coma cluster. This percentage does not vary strongly across the field, and range typically from 14 to $23 \%$. An explanation could be the presence of more distant concentrations, distinct from Coma, between $z \sim 0.16$ and 0.18 (see A06a) and spread over the whole Coma field. To check this hypothesis, we translated the Coma cluster RS to a 0.17 redshift using the elliptical galaxy synthetic models of Coleman et al. (1980). The new RSs obtained are shown in Fig. 17 as the green/grey lines and reasonably correspond to the locations where an excess of red objects is detected.

Finally, by simply counting the number of spectroscopic redshifts available between $z \sim 0.16$ and 0.18 in the Coma field, we find a number of redshifts equal to $\sim 15 \%$ of the total number of redshifts inside the Coma cluster itself, in good
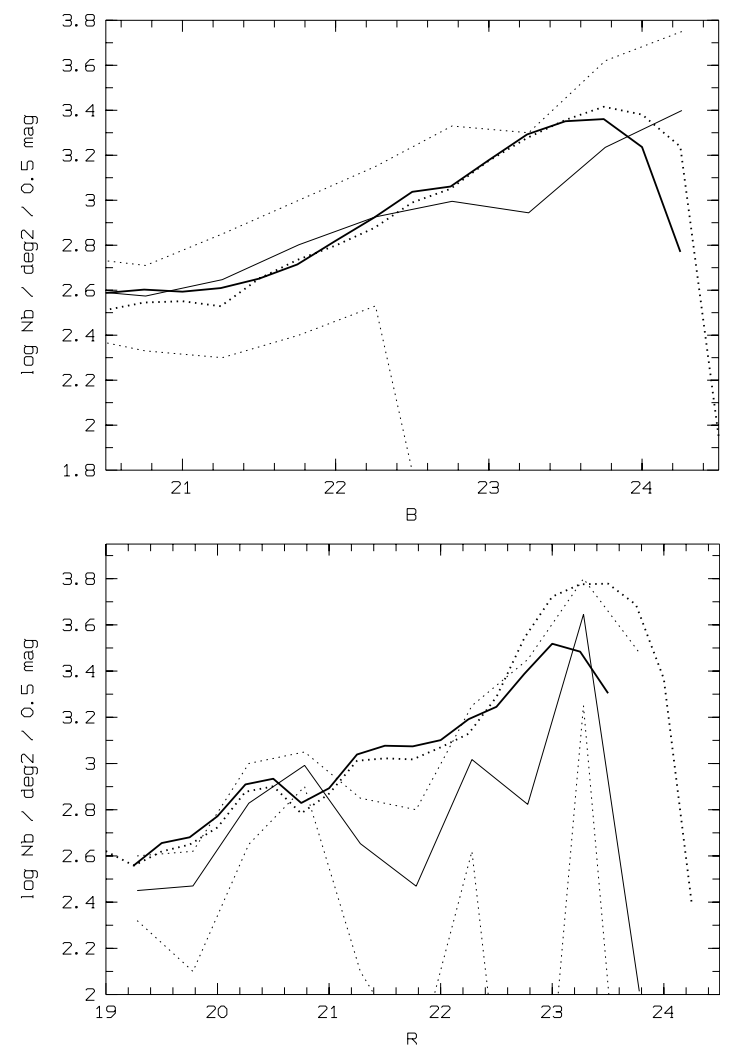

Fig. 16. Same as Fig. 15: comparison with Trentham (1998); from top to bottom: $B$ - and $R$-bands.

agreement with the percentages of very red objects previously found. These galaxies spectroscopically located at $z \sim 0.17$ extend all across the field and are probably related to a distant filament on the line of sight that we will not study here. This background structure is probably the same as the one at $\sim 49000 \mathrm{~km} \mathrm{~s}^{-1}$ quoted by Gutiérrez et al. (2004), with a velocity dispersion of $1000 \mathrm{~km} \mathrm{~s}^{-1}$. In conclusion, the population of objects fainter than $R=19.5$ seen in excess in the Coma field compared to the empty fields is well explained by the contributions of objects inside the Coma cluster globally following the bright Coma cluster galaxy RS and of a population of very red objects at $z \sim 0.17$ that we will not consider in the following.

\subsection{Location of Coma cluster objects in the $B-R / R$ relation relatively to the $R S$ of bright galaxies}

Looking at Coma cluster objects in more detail, in Fig. 17 we can also see that there are two populations close to but outside of the RS of bright galaxies: one is redder (distinct from the $z \sim 0.17$ very red population previously quoted) and the other bluer, with distributions depending on the location in the field. We will refer to these two populations in the following as the red and blue galaxies. The south zone exhibits significant (see Fig. 18) red object concentrations at $R \sim 22-23$, while the north zone seems to show fewer red objects. We also have a significant blue population in the north. This blue concentration is also visible in the south regions, but is not always significant. We also note some regions in the south where empty fields galaxy populations dominate the Coma line of sight. This can be due to local overestimate of the background galaxy population, but is perhaps also due to merging/disruption events inside the Coma cluster, removing galaxies from the counts. 
There is a variation of colors as a function of location in the cluster. This is visible on the mean slope of the CMR as a function of magnitude (see Fig. 17). The south shows a mean negative slope of $-0.044 \pm 0.014$ (characterizing the whole Coma cluster population, not only the early-type galaxies included in the bright CMR RS), while the North exhibits a slope of $-0.084 \pm$ 0.031 .

\subsection{Contribution of intergalactic globular clusters?}

As explained earlier, our data are by definition free of GCs still linked to giant galaxies. However, we may still have detected intergalactic GCs or GCs attached to faint galaxies even if this contribution is probably minor (Marín-Franch \& Aparicio 2003). Nevertheless, the brightest magnitudes of GCs are not bright enough to completely account for the red objects between $R=22$ and 23 in the south field. GCs could contribute to the excesses of blue objects in the north fields at $\mathrm{R}$ fainter than 23 and $B-R \sim 0.7$, but there should be only a few GCs existing with such blue colors (e.g., Rhode \& Zepf 2004) at these magnitudes, and this contribution should be minor. In conclusion, GCs do not allow us to account for the red and blue object excesses statistically detected in the Coma cluster. A significant part of these blue objects must therefore be galaxies.

\section{Inferences from the Coma LF on the cluster building up scenario}

\subsection{Previous Coma cluster literature luminosity functions and environmental effects}

In their pioneering work, Bernstein et al. (1995) analyzed the Coma LF in a small but very deep field close to NGC 4874 and found a faint end Schechter slope of $\alpha=-1.42$. However, the field was too small to show evidence for large-scale environmental effects. Lobo et al. (1997) were the first to derive relatively deep $V$-band galaxy LFs in various regions of the Coma cluster. They showed that the faint end slope of the LF was flatter in the central regions $(\alpha=-1.51$ to -1.58$)$ and steeper in the outskirts (as steep as -1.88 ), with error bars of \pm 0.10 . They proposed a dynamical interpretation based on the recent history of the two subclusters surrounding the giant galaxies NGC 4874 and NGC 4889: when these two groups fell into the central region of Coma, the large number of dwarf galaxies initially present may have rapidly been accreted by the largest ones, therefore flattening the faint end slope of the LF. Trentham (1998) confirmed that the faint end slope of the overall LF could be as steep as -1.7 ; however, he did not find any difference in the faint end slope of the LF between the inner $(r<200 \mathrm{kpc})$ and outer $(r>200 \mathrm{kpc})$ regions. Neither did Iglesias-Páramo et al. (2003), who only report a steepening towards the outskirts significant at the $1 \sigma$ level. Beijersbergen et al. (2002) found that the LF slope was steeper towards the blue $(\alpha=-1.54,-1.32$, and -1.22 in $U, B$, and $r$, respectively, for the full sample). They also found a stronger steepening in the $U$-band toward the cluster edges. Simultaneously, Andreon \& Cuillandre (2002) observed Coma in smaller but somewhat deeper fields and also found that the slope and shape of the LF was dependent on color.

However, all these studies were not well adapted in terms of combined depth, area, and spectral coverage to properly investigate environmental effects in the Coma cluster. They were able to sample different cluster regions (but most of the time assuming an axisymmetric geometry that is not valid here), or different galaxy magnitude classes, or LFs in different colors, but never all together. Our data allow us to reach such goals simultaneously. For example, the Coma cluster building process proposed in Adami et al. (2005b) allows us to interpret our results in terms of infalls onto the cluster coming from well defined directions: northeast-east, northwest-west, and south-west. We will now show that the GC distribution and luminosity function shapes are in good agreement with this framework.

\subsection{Spatial distribution of GCs}

The high fraction of GCs found close to NGC 4874 (see also Marín-Franch \& Aparicio 2003) can be explained by assuming that it is the oldest cluster dominant galaxy, located at the bottom of the original cluster potential well. When the GC parent galaxies passed through the inner cluster core (where the tidal interactions are the strongest), part of their GCs may have been expelled, leading to a high concentration of GCs in that zone. The other region showing atypical GC percentages is in the north part of the cluster (subfield 15), where about half of the objects between $V=23.75$ and $V=24.25$ have colors consistent with GCs. This region does not contain major galaxies and a possible explanation (assuming that the GC percentage is correct) is that a galaxy passing through has lost part of its GC population.

\subsection{Infall directions and luminosity functions}

The literature on Coma cluster LFs sometimes seems to lead to contradictory conclusions, probably because these studies usually assume an axisymmetric geometry that is not correct. As we show in the present paper, the variations are more complex and follow an overall nearly north/south symmetry and some peculiar processes related to the bright galaxies. The south regions showing quite flat LFs are also those located on the main field infall directions, symbolized for example by the infalling NGC 4839 group (not sampled by our data), see Lobo et al. (1997). The north regions are not subject to major known infalls and show steeply rising LFs. The LF shapes could then be related to the presence or not of directions of infall: the north regions being only poorly fed by external infalls, we search for the explanation in the south infalls.

First, galaxies infalling from the south can enhance the north population after having crossed the entire cluster. We would need about 1500 galaxies coming from the south to be located in the north region. This number is the difference between the north and south LFs between $R=18$ and 22 (the faintest magnitude in the south region where the LF can still be computed with our data). This number is plausible given the infall rate given in the following (see Adami et al. 2005b), but we clearly need simulations to assess this process.

Second, the infall of galaxies from the south can also diminish the south galaxy population by several processes:

- Merging between faint galaxies to form brighter objects cannot work because in this scenario we should have a significant overabundance of bright galaxies in the south compared to the north and this is not the case.

- Merging of faint galaxies with brighter objects could be another explanation, since this process is independent of galaxy type and therefore of galaxy color. Figure. 13 shows that all the regions in the south exhibit nearly flat LFs for both blue and red objects, so the process removing faint galaxies from the counts has to be exterior to the galaxies, such as for example a fusion scenario. 

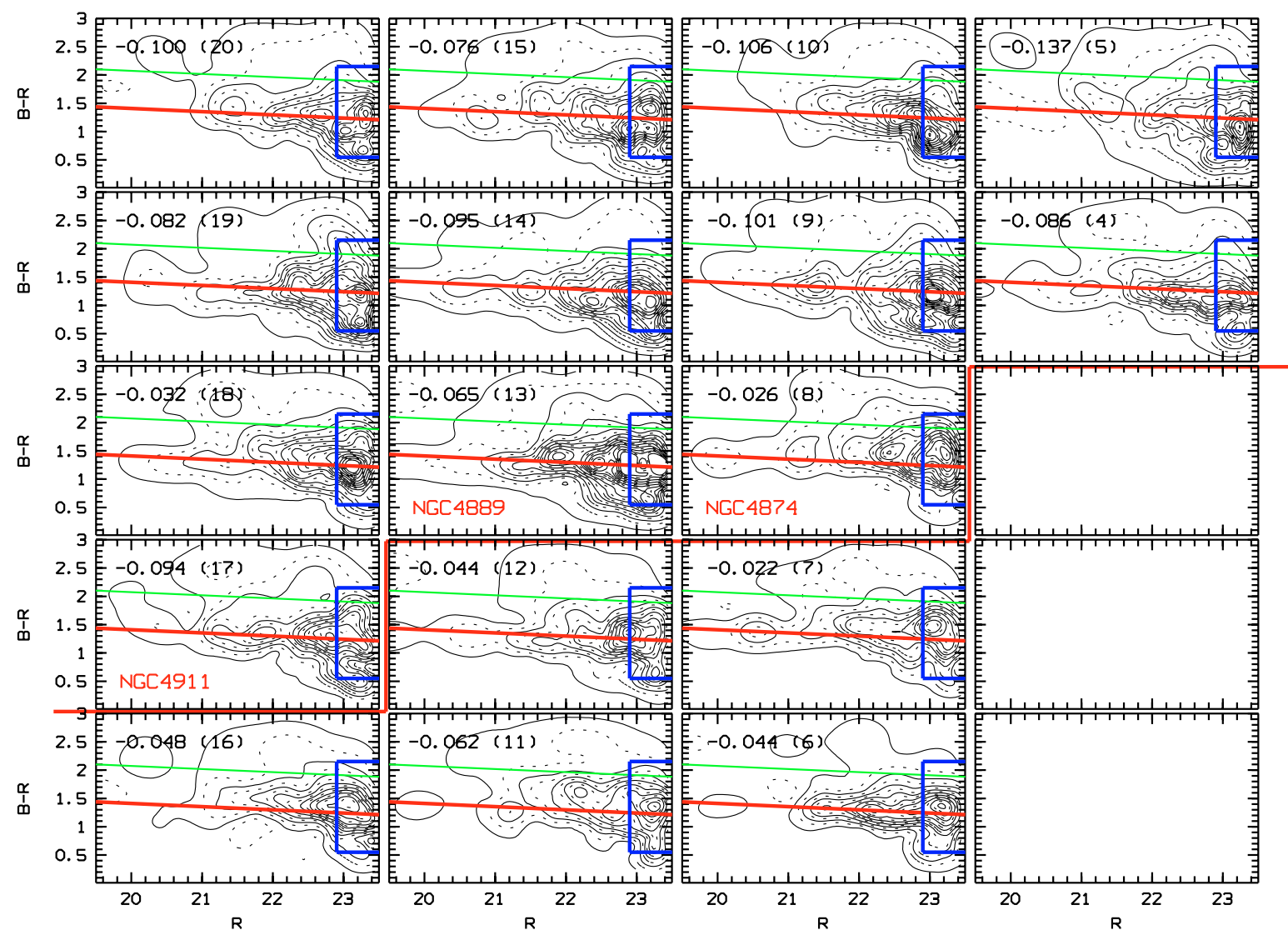

Fig. 17. CMR for different locations in the Coma cluster. North is top and east left. Locations of the three main galaxies (NGC 4874, NGC 4889, and NGC 4911) are shown. The red/black inclined line is the RS computed in A06a using bright galaxies with spectroscopic redshifts inside the Coma cluster. The green/grey inclined line is the same RS shifted to $z=0.17$ using synthetic elliptical galaxy templates. The black/blue rectangles at faint magnitudes are the locations where intergalactic globular clusters can pollute the galaxy sample. The red broken line delineates the north and south fields. We also give the mean CMR slope along with the subfield number for each subfield.

Adami et al. (2005b) have indeed shown that a mass between 0.4 and $2.0 \times 10^{14} M_{\odot}$ originating from field galaxy groups has fallen onto the Coma cluster in a Hubble time. Assuming that all this matter was constituted of galaxies, we then have a strict upper value of the number of galaxies captured by Coma. This represents a maximum of $\sim 4000$ galaxies of $0.1 M^{*}$ (using Mamon 2000).

Mamon (2000) gives an estimate in a Hubble time of the fusion number of $0.1 \mathrm{M}^{*}$ galaxies with lower mass objects as a function of distance to the cluster center. For simplicity we will use the values given at $0.05,0.1,0.2$, and 0.3 virial radii.

Multiplying these numbers by the maximal number of infalling galaxies, we then have estimates of the numbers of objects fainter than $0.1 M^{*}$ accreted by $0.1 M^{*}$ galaxies as a function of distance to the cluster center. By comparing these numbers with the density of lacking galaxies in the south relative to the north, we find that the proposed merging process is only able to explain $\sim 25 \%$ of the lacking galaxies at 0.05 virial radius, $\sim 5 \%$ at 0.1 virial radius, and less than $1 \%$ at 0.2 and 0.3 virial radius. Therefore this process can have a significant effect close to the cluster center, but is overall inefficient above 0.1 virial radius.

- Destruction of faint galaxies by tidal effects cannot act alone because we do not detect any significant large-scale diffuse light source in the south (see Adami et al. 2005a), far from the dominant cluster galaxies. It could possibly act locally, but is not sufficient to explain the entire faint galaxy deficit.
We also estimated the possible number of faint galaxies removed by this process using Mamon (2000) and Adami et al. (2005b). Counting the number of strong tidal encounters, we found that this effect is at maximum $1 / 10$ th of the previous process (merging of faint galaxies with brighter objects). It is therefore negligible.

- A last explanation would be the inhibition of faint galaxy formation in the south compared to the north. We know for example that faint galaxies can be formed by tidal encounters (e.g. Bournaud et al. 2003) inside the cluster. An example of such formed galaxies could be the blue low surface brightness galaxies of Adami et al. (2006b). This process could be inhibited by modest tidal forces induced by infalling galaxies. Here we show a marginal overabundance of blue low surface brightness galaxies in the north subfields compared to the south. Assuming the same overabundance for all faint galaxies, this can, however, only explain $\sim 10 \%$ of the galaxies lacking in the south.

In conclusion, the merging of faint galaxies with brighter objects and faint galaxy formation inhibition in the south can only explain part of the south galaxy deficit compared to the north. Another explanation, which still has to be verified via numerical simulations, is an accumulation in the north of faint galaxies coming from the south. 

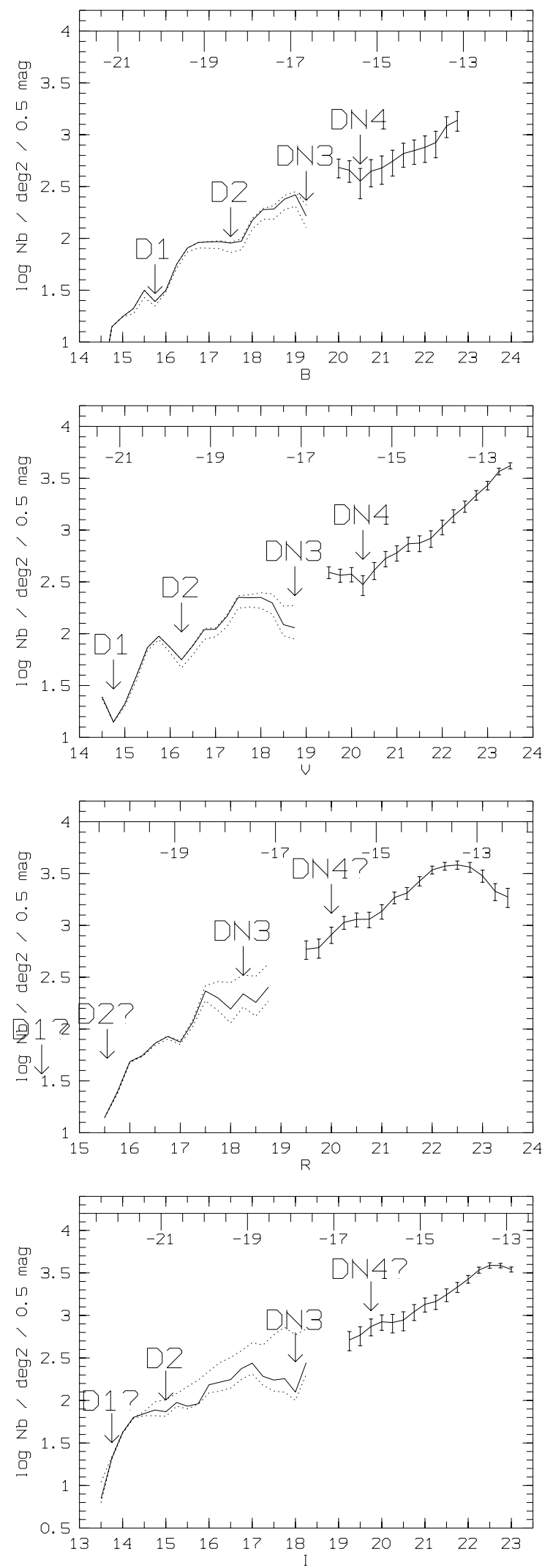

Fig. 19. $B V R I$ LFs for the north-northeast area. We give both apparent and absolute magnitudes. The continuous line on the left half is the LF built from spectroscopy with its $1 \sigma$ uncertainty envelope (dotted lines). The continuous line to the right is the LF built from statistical subtraction without any use of color information. Error bars come from the methods explained in the text. The position of the dips in all colors is also given.
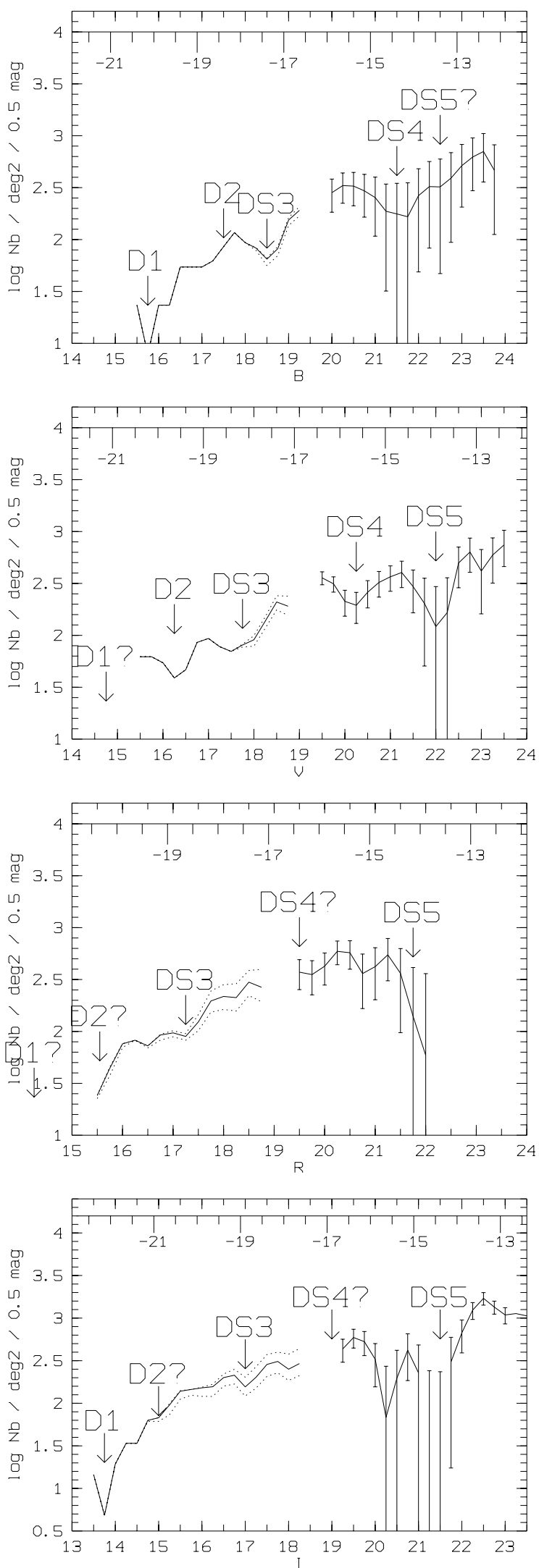

Fig. 20. Same as Fig. 19 but for the south-southwest region.

\subsection{What happens around the dominant galaxies NGC 4874} and NGC 4889?

The LF steeply rises around NGC 4874 in $I$ and $R$, is flatter in $V$, and quite flat in $B$. Moreover, most of the objects around 
NGC 4874 are red, except at very faint magnitudes where blue and red objects have the same contribution. We must find the process(es) explaining such behavior. We have already shown that a non-negligible part of the objects around NGC 4874 have colors consistent with those of GCs, i.e., are red. NGC 4874 is also probably the oldest dominant galaxy in the Coma cluster (e.g., Adami et al. 2005b or Neumann et al. 2003), therefore located at the bottom of the cluster potential well and affected by very strong environmental effects (e.g. Odell et al. 2002). This region is very dense and populated by the oldest Coma cluster galaxies which have probably already used up their gas reservoir, since they are now quite quiescent, from the star-forming point of view. This should explain why they appear red and are not easily detected in the blue band.

Around NGC 4889 the LF steeply rises in all the observed bands and there are no particularly red objects in this region. This is confirmed by the low percentage of objects detected with colors consistent with GCs. Moreover, if NGC 4889 has a recent history in the Coma cluster, its satellite galaxies have not yet had time to stop their star formation due to cluster environmental effects.

\subsection{Large-scale dips in the Coma cluster luminosity functions}

We see several dips in the LFs across the Coma cluster field of view, making the LFs clearly not purely Schechter-like. Are these dips real or due to bias in the statistical subtraction method? These dips could come from empty field oversubtractions due to the existence of structures not present along the Coma cluster line of sight. However, they appear when using both the F02 or F10 comparison fields, so they are probably real. They concern galaxies within a relatively narrow magnitude range. We have no explanation for this, but it probably indicates that the dips are linked to physical processes involving only certain galaxy populations.

For simplicity, we only discuss the dips seen in Figs. 19 and 20. As previously discussed, at zero order a dip shows that galaxies have been removed from the counts. In a simple model, these removals can be due either to the disappearance of galaxies (through merging or destruction visible in all the bands) or to galaxy faintening (star-forming activity inhibition mainly visible in the blue bands). We note that a brief star-forming activity enhancement during the merging or the destruction is also possible, inducing a bump in the blue bands and a dip in the red bands.

We chose to first search for dips in the $B$ - or $V$-bands. Later, we checked if these dips were also visible in the $R$ - and $I$-bands. We mainly detected two dips visible both in the north and south fields: D1 and D2. We also detected two dips visible in the north field (DN3 and DN4) and three in the south field (DS3, DS4 and DS5). The dip characteristics are summarized in Table 6. The results suggest that the southern region is probably subject to the global disappearance of galaxies and not to simple starforming inhibition (because all the dips observed in the south are visible in all the bands), while the northern region shows both processes.

\section{CMR and the Coma cluster history}

It is remarkable that most of the faint Coma cluster galaxies overall follow the bright galaxy RS. This means that a large fraction of the faint Coma cluster galaxies have evolutionary paths similar to bright cluster galaxies in terms of metallicities. However,
Table 6. Dips seen at large scale across the Coma field of view.

\begin{tabular}{lll}
\hline \hline & Magnitude & Origin \\
\hline D1 & $B \sim 15.75$ & Bright elliptical mergings \\
D2 & $B \sim 17.5$ & Disappearance of red elliptical-like galaxies \\
& & Potentially detected in Andreon \& Pelló (2000) \\
DN3 & $B \sim 19.25$ & Disappearance of spiral galaxies \\
DN4 & $B \sim 20.5$ & Late-type spiral star formation inhibition \\
DS3 & $B \sim 18.5$ & Disappearance of bright spiral galaxies \\
& & Potentially detected in Lobo et al. (1997) \\
DS4 & $B \sim 21.5$ & $?$ \\
DS5 & $V \sim 22$ & Disappearance of faint spiral galaxies \\
\hline
\end{tabular}

if we look more carefully at the Coma cluster galaxy distribution in the CMR, we see blue and red objects close to the bright galaxy RS.

\subsection{Blue objects in the CMR}

These objects, detected over nearly the whole Coma cluster field (Fig. 17), except perhaps around NGC 4874 and not always significantly in the south, have colors typical of late-type galaxies suggesting that they still have a significant star-formation activity. As we already discussed, they are only marginally explained by GCs, and are probably galaxies. We suggest that some of these objects can be recently formed galaxies in the Coma cluster. This is supported by the fact that we have detected a large population of faint $(R<21)$ and low surface brightness galaxies in the Coma cluster with atypically blue colors (Adami et al. 2006b). We suggested in this paper that these objects originated from external material expelled from brighter late-type galaxies. Significant concentrations of these faint blue low surface brightness galaxies are detected in several places across the Coma cluster: mainly in the western part of the cluster and around NGC 4889. They also have the right $B-R$ colors and the right $R$ magnitudes to explain at least some of the blue objects visible in Fig. 17. However, concentrations of blue Coma cluster objects are also detected where there is no significant concentration of faint blue low surface brightness galaxies. These galaxies are therefore not the only explanation for our blue objects.

\subsection{Red objects in the CMR}

Contrary to the blue galaxies, faint red objects close to the RS can be at least partially explained by GCs. However, we also detect red objects that are too bright to be GCs, mainly in the south area and around NGC 4874. Their colors suggest that they have a low star-formation activity. They could also be similar to the nucleated dwarfs studied by Rakos \& Schombert (2004) that were found to be redder than bright elliptical galaxies.

\subsection{Slope of the $C M R$}

We computed a slope of -0.045 for the RS bright early-type galaxies in A06a. This slope is very similar to the CMR slope (for all available galaxy types) in the south regions. This is a way to show that the galaxy populations in the south fields are mainly similar to bright red early-type galaxies. In the north, the negative slope is steeper. The universal metallicity-mass relation found by Terlevich et al. (2001) for the bright galaxies is then not valid for the whole faint galaxy population. This expresses the predominance of blue objects that followed different evolutionary paths in the north fields compared to bright ellipticals. 
More precise estimates in terms of ages and metallicities are difficult because we do not know which part of the observed characteristics are coming from internal galaxy physics and which from external processes due to environmental effects. We will therefore not push this discussion further.

\section{Conclusions}

In this paper, we have analyzed the deep galaxy LF in the $B-, V$-, $R$-, and $I$-bands and the faint $B-R / R C M R$ for the Coma cluster. We applied a single Schechter law to model our LFs. To sample possible environmental effects on the LF, we derived twenty such functions in $10 \times 10 \mathrm{arcmin}^{2}$ areas. We found a dichotomy between steeply rising LFs in the north-northeast Coma region, which appear to be due to red/early-type galaxies at bright magnitudes, and to blue/late-type objects at very faint magnitudes, and much flatter LFs in the south-southwest region, both for all objects and for blue/late-type or red/early-type galaxies. This result is valid for all photometric bands and all observation epochs.

We also found that the LFs differ around the main Coma cluster galaxies and groups. LFs in the NGC 4889 field are steeply rising in all photometric bands, while around NGC 4874 they are rising moderately in $B$ and $V$ and steeply in $R$ and $I$, with a maximum reached around $R \sim 23$ and $I \sim 22.5$. Around NGC 4911 , the LF is moderately rising in $B$ and $V$ up to $B \sim$ 23.75 and $V \sim 23.5$ and oscillating and rising up to $R \sim 23$ and over the whole $I$ magnitude range.

We have discussed the observed shapes of the LFs in various regions in the framework of the building up process that we have proposed in a previous paper (Adami et al. 2005b). In this scenario, several infalls onto the Coma cluster are taking place, coming from three directions: northeast-east, northwestwest, and south-west. NGC 4874 is the original cluster dominant galaxy, while NGC 4889 experienced a later capture. How does this match our observations?

The GC expelling scenario explains the high fraction of GCs found close to NGC 4874 quite well. The south-southwest regions showing quite flat LFs are also those which are directly located on the main field infall directions (in particular the infalling NGC 4839 group). The observed paucity of faint galaxies in the southern region is only partially explained by the fact that faint galaxies may have been swallowed by relatively bright galaxies on their way to the cluster center. Rather, north counts could be enhanced by these south infalls to explain their steeply rising LFs (the north part has a LF slope significantly different from the field, e.g., Ilbert et al. 2005). However, we can note that faint galaxies are blue/late-type while bright galaxies are red/early-types in the north. This suggests that these blue and faint galaxies could also partially be created in the cluster from material expelled from the envelopes of spiral-like parent galaxies. We have detected several dips in the LFs in different areas of the cluster and shown that some of them are probably due to mergings/fusions, while others are probably due to the inhibition of star-formation activity by cluster environmental effects.

Finally, we have also clearly detected a RS down to $R=23.5$, close to the bright galaxy RS computed in A06a. This shows that most of the faint Coma cluster galaxies have probably followed the same evolutionary path as the bright ellipticals in terms of mass-metallicity relation. However, part of the faint cluster population followed a different evolution on the blue and red sides. We suggest that blue objects present over the whole Coma cluster field are partially due to recent galaxy formation from material expelled from late-type galaxies. It is particularly interesting to note that the CMR extends over such a large range of magnitudes, thus making it a very useful tool for several purposes, such as selecting galaxies with a high probability of belonging to a cluster from imaging data only. In the near future, we intend to compute and discuss the Coma cluster luminosity functions based on photometric redshifts as soon as we will have acquired the $U$-band data lacking for our survey (see A06a).

Acknowledgements. The authors thank the referee for useful and constructive comments. The authors are grateful to the CFHT and Terapix teams for their help and to the French PNG, CNRS for financial support. The authors thank B. Lanzoni and G. Mamon for useful discussions and for providing their numerical simulations.

\section{References}

Adami, C., Ulmer, M. P., Durret, F., et al. 2000, A\&A, 353, 930

Adami, C., Slezak, E., Durret, F., et al. 2005a, A\&A, 429, 39

Adami, C., Biviano, A., Durret, F., \& Mazure, A. 2005b, A\&A, 443, 17

Adami, C., Picat, J. P., Savine, C., et al. 2006a, A\&A, 451, 1159 (A06a)

Adami, C., Scheidegger, R., Ulmer, M. P., et al. 2006b, A\&A, 459, 679

Andreon, S., \& Pelló, R. 2000, A\&A, 353, 479

Andreon, S., \& Cuillandre, J.-C. 2002, ApJ, 569, 144

Baum, W. A. 1959, PASP, 71, 106

Beijersbergen, M., Hoekstra, H., van Dokkum, P. G., \& van der Hulst, T. 2002, MNRAS, 329, 385

Bernstein, G. M., Nichol, R. C., Tyson, J. A., Ulmer, M. P., \& Wittman, D. 1995, AJ, 110, 1507

Bertin, E., \& Arnouts, S. 1996, A\&ASS, 117, 393

Biviano, A. 1998, Untangling Coma Berenices: A New Vision of an Old Cluster, Proceedings of the meeting held in Marseilles, France, June 17-20, 1997, ed. A. Mazure, F. Casoli, F. Durret, \& D. Gerbal (Word Scientific Publishing Co Pte Ltd), 1, Also:

http://nedwww.ipac.caltech.edu/level5/Biviano/frames.html Biviano, A., Durret, F., Gerbal, D., et al. 1996, A\&A, 311, 95

Bournaud, F., Duc, P. A., \& Masset, F. 2003, A\&A, 411, L469

Coleman, D. G., Wu, C. C., \& Weedman, D. W. 1980, ApJS, 43, 393

Durret, F., Adami, C., \& Lobo, C. 2002, A\&A, 393, 439

Edwards, S. A., Colless, M., Bridges, T. J., et al. 2002, ApJ, 567, 178

Fukugita, M., Shimasaku, K., \& Ichikawa, T. 1995, PASP, 107, 945

Gazelle, F., Robin, A., \& Goidet-Devel, B. 1995, Vistas in Astronomy 39, 105

Geller, M. J., Diaferio, A., \& Kurtz, M. J. 1999, ApJ, 517, L23

Gutiérrez, C. M., Trujillo, I., Aguerri, J. A. L., Graham, A. W., \& Caon, N. 2004, ApJ, 602, 664

Hansen, S. M., McKay, T. A., Wechsler, R. H., et al. 2005, ApJ, 633, 122

Hoyle, F., \& Vogeley, M. S. 2004, ApJ, 607, 751

Huang, J. S., Cowie, L. L., \& Gardner, J. P. 1997, ApJ, 476, 12

Iglesias-Páramo, J., Boselli, A., Gavazzi, G., Cortese, L., \& Vílchez, J. M. 2003, A\&A, 397, 421

Ilbert, O., Tresse, L., Zucca, E., et al. 2005, A\&A, 439, 863

Kodama, T., \& Arimoto, N. 1997, A\&A, 320, 41

Lanzoni, B., Guiderdoni, B., Mamon, G. A., Devriendt, J., \& Hatton, S. 2005, MNRAS, 361, 369

Le Fèvre, O., Vettolani, G., Garilli, B., et al. 2005, A\&A, 439, 845

Lobo, C., Biviano, A., Durret, F., et al. 1997, A\&A, 317, 385

Mamon, G. A. 2000, ASPC, 197, 377 [arXiv:astro-ph: 9911333]

Marín-Franch, A., \& Aparicio, A. 2003, ApJ, 585, 714

Mazure, A., Proust, D., Mathez, G., \& Mellier, Y. 1988, A\&ASS, 76, 339

McCracken, H. J., Radovich, M., Bertin, E., et al. 2003, A\&A, 410, 17: MC03

Milne, M. L., Pritchet, C. J., Poole, G. B., et al. 2007, AJ, in press

Neumann, D. M., Lumb, D. H., Pratt, G. W., \& Briel, U. G. 2003, A\&A, 400, 811

Odell, A. P., Schombert, J., \& Rakos, K. 2002, AJ, 124, 3061

Pickles, A. J. 1998, PASP, 110, 863

Rakos, K., \& Schombert, J. 2004, AJ, 127, 1502

Rhode, K. L., \& Zepf, S. E. 2004, AJ, 127, 302

Sandage, A. 1972, ApJ 176, 21

Sarazin, C. L. 1986, Rev. of Mod. Phys., 58, 1

Savine, C. 2002, Ph.D. Thesis, Université de Provence

Schlegel, D. J., Finkbeiner, D. P., \& Davis, M. 1998, ApJ, 500, 525

Terlevich, A. I., Calswell, N., \& Bower, R. G. 2001, MNRAS, 326, 1547

Trentham, N. 1998, MNRAS, 293, 71

Yee, H. K. C., Gladders, M. D., \& López-Cruz, O. 1999, ASPC, 191, 166 
C. Adami et al.: Spatial variations of the Coma luminosity functions and color-magnitude relations, Online Material p 1

\section{Online Material}


C. Adami et al.: Spatial variations of the Coma luminosity functions and color-magnitude relations, Online Material p 2
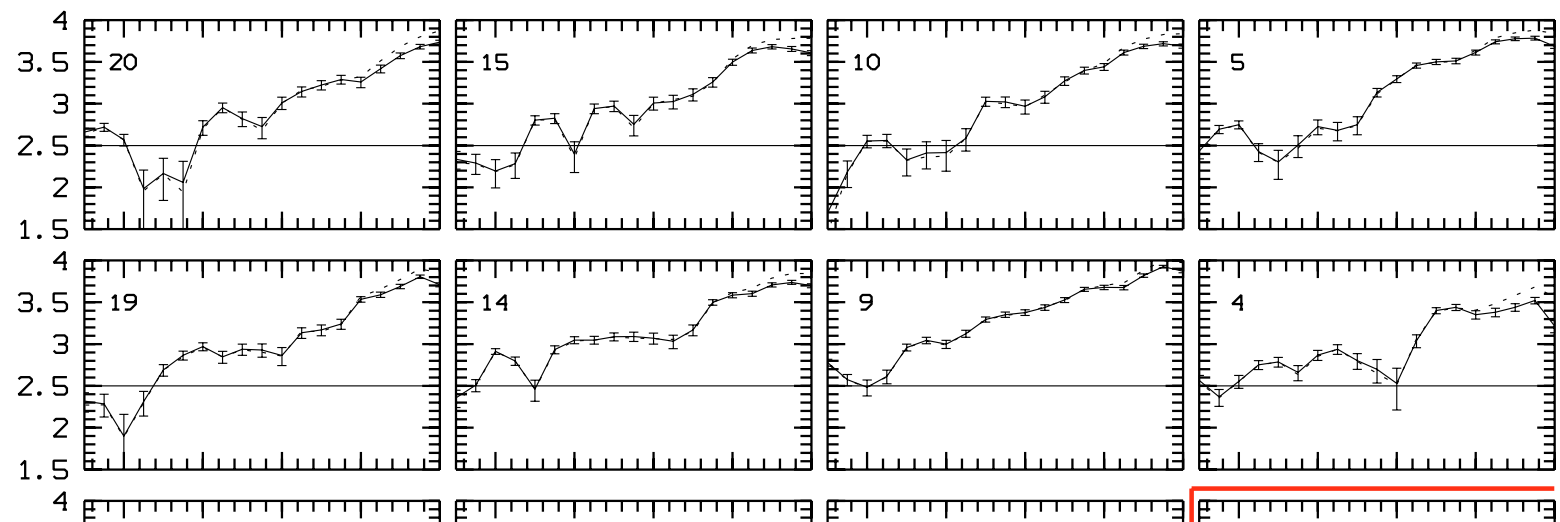

3.5 年 18

3.5

2.5 2

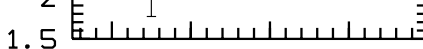
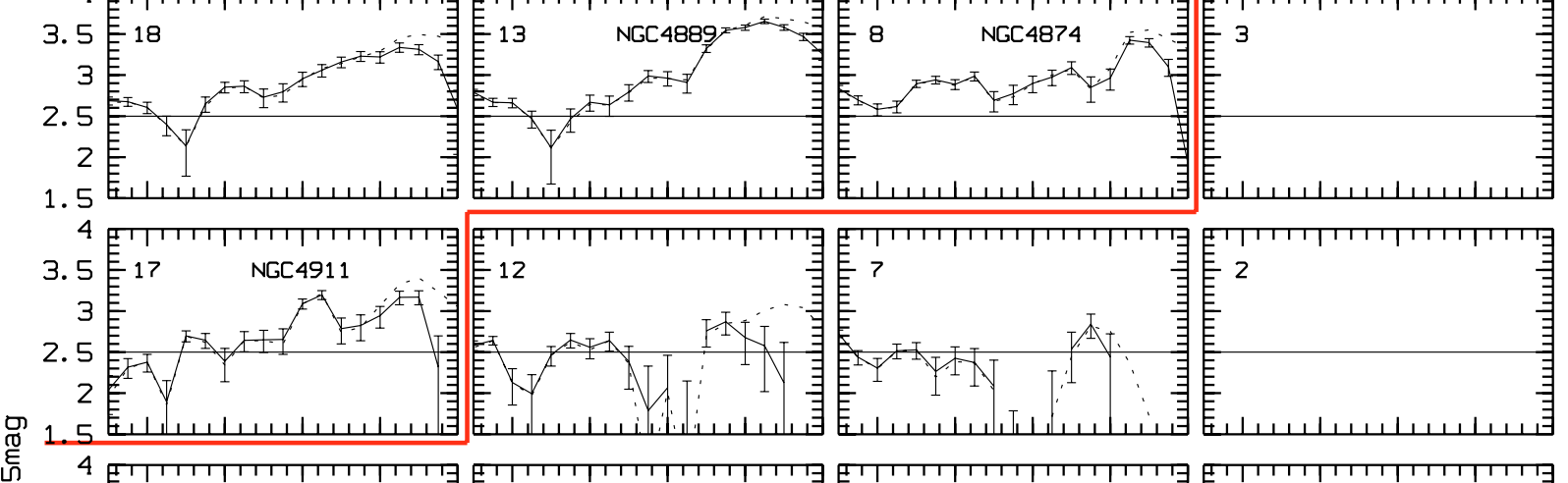

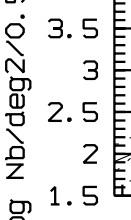

믐
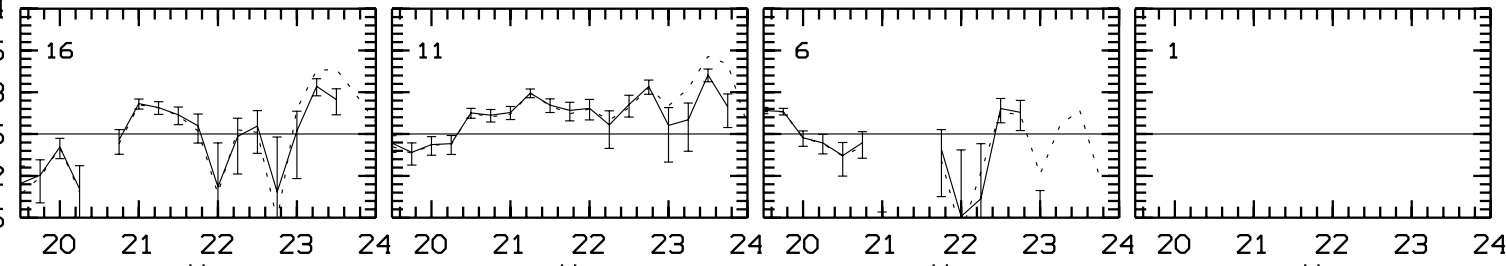

Fig. 10. Same as Fig. 9 for the $V$-band. 
C. Adami et al.: Spatial variations of the Coma luminosity functions and color-magnitude relations, Online Material p 3
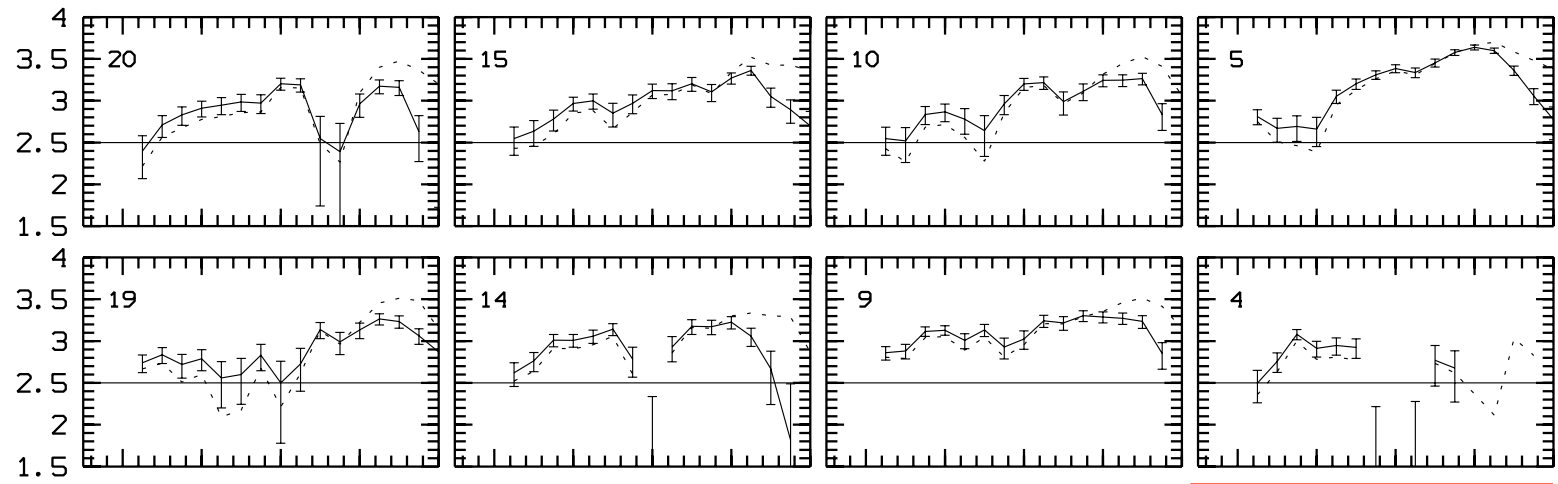

4 सागा

3.5 年 18

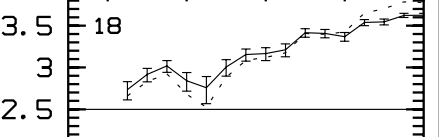

2

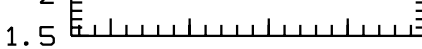
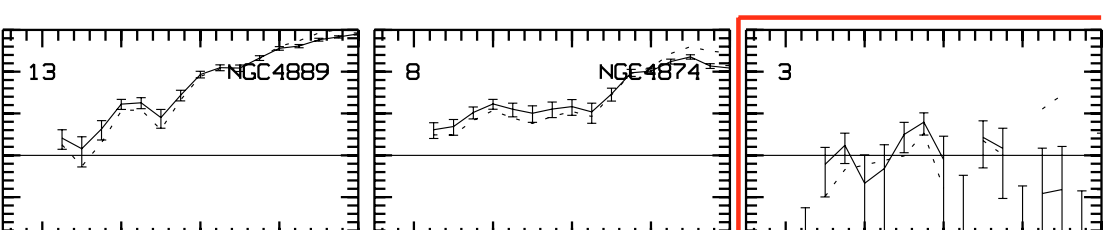

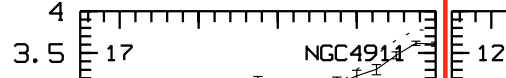

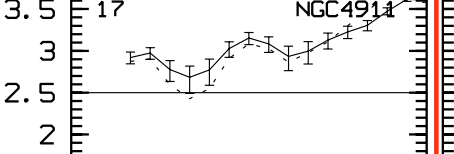

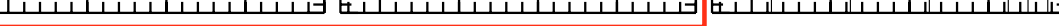

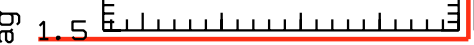
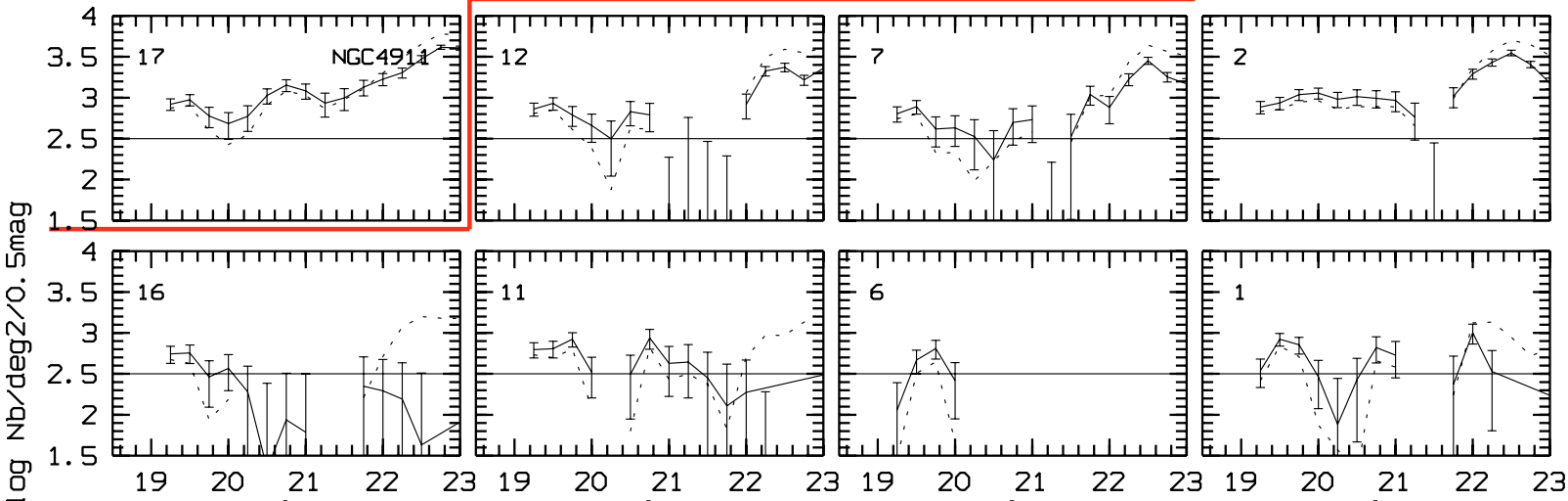

Fig. 12. Same as Fig. 9 for the $I$-band. 
C. Adami et al.: Spatial variations of the Coma luminosity functions and color-magnitude relations, Online Material p 4
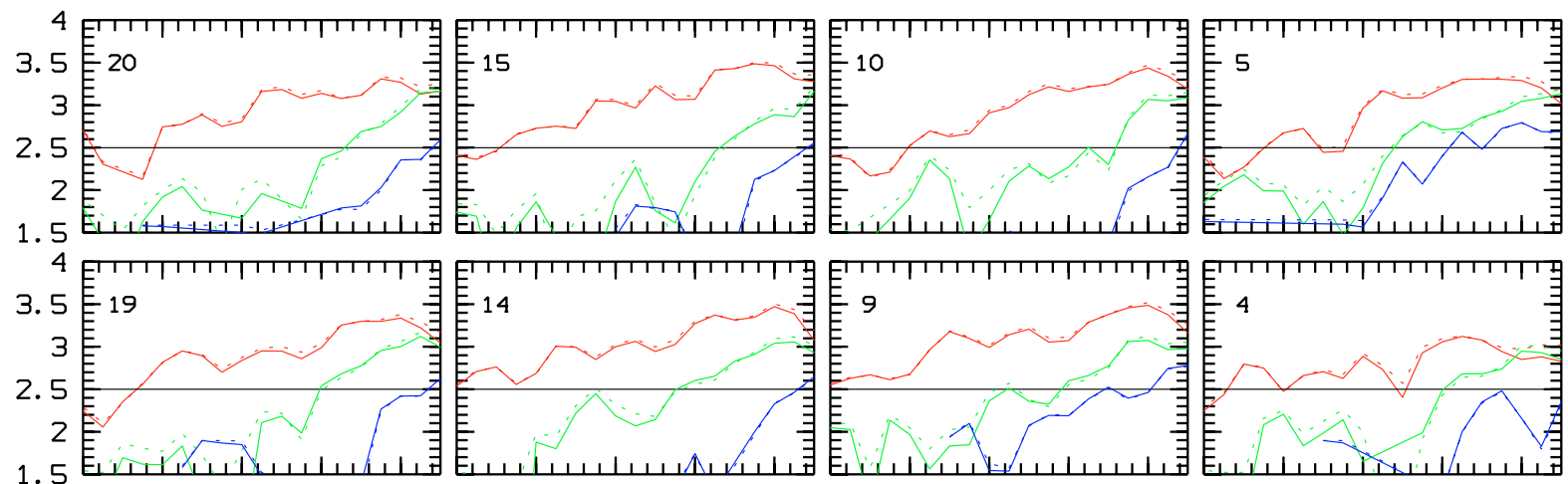

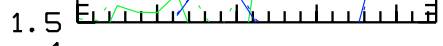
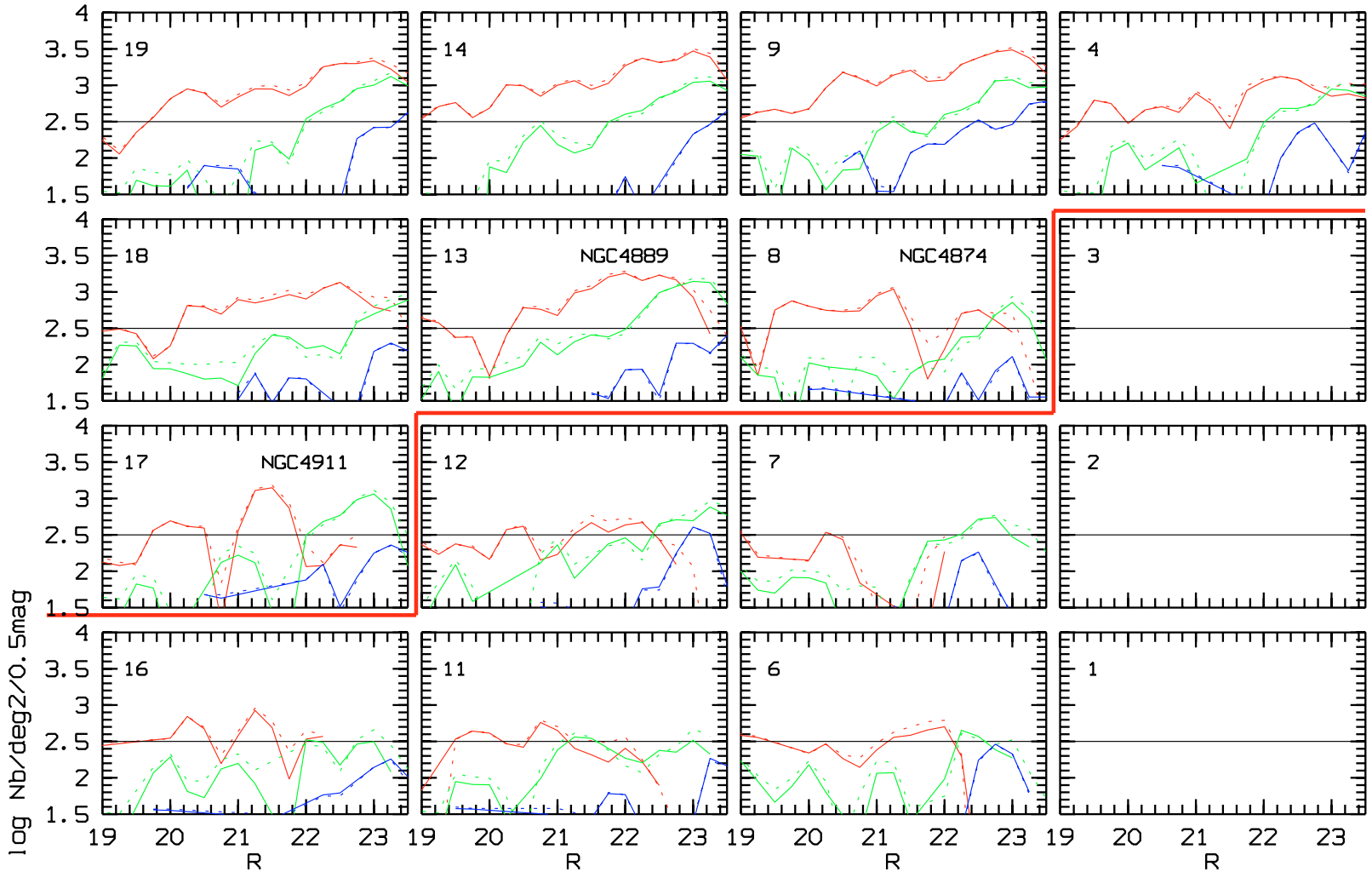

Fig. 14. Same as Fig. 9 for early type galaxies (black/red lines), early spiral galaxies (light grey/green lines), and late spiral galaxies (heavy black/blue lines). Error bars are not shown for clarity. 
C. Adami et al.: Spatial variations of the Coma luminosity functions and color-magnitude relations, Online Material p 5


Fig. 18. Same as Fig. 17 with different colors according to significance levels of densities given in Fig. 17. Black shaded regions: between 1 and $3 \sigma$ levels; red/light-black shaded areas: between 3 and $4 \sigma$ levels; green/grey shaded regions: above $4 \sigma$ levels. The white areas embedded in colored areas are significant at less than $1 \sigma$ (or even regions where empty fields dominate the Coma line of sight population). The blue inclined thick lines show the red sequence of bright galaxies computed in A06a. 